\title{
Periodicidade para uma classe de Equações Diferenciais Retardadas no Plano
}

\author{
Maria Elisa Quiroga
}

Dissertação apresentada ao Instituto de Ciências Matemáticas de São Carlos, da Universidade de São Paulo, para obtenção do Título de Mestre em Matemática

Orientador: Prof. Dr. Plácido Zoega Táboas

São Carlos

1992 
A Deus, sem o qual nada seria possível. 
Aos meus pais (in memorian)

Ao meu filho Rafael 


\section{Agradecimentos}

Ao Prof. Dr. Plácido Z. Táboas, pela segurança na condução dessa minha iniciação à pesquisa. A confiança, o entusiasmo e a dedicação irrestrita constituem palavras de denotação fraca perante todo o relacionamento humano que perpassou por sua orientação.

Aos professores do Departamento de Matemática, do Instituto de Ciências Matemáticas de São Carlos e colegas de mestrado, pelo ótimo ambiente de estudo, pelo apoio, pelo incentivo e pelas conversas que proporcionaram momentos de iluminação, sensibilidade e grandeza.

A todos aqueles que direta ou indiretamente colaboraram para a realização deste trabalho.

À CAPES, pelo apoio financeiro parcial. 


\begin{abstract}
We are concerned with periodicity results for the planar delay-differential equation

$$
x^{\prime}(t)=-x(t)+\alpha F(x(t-1)), \quad \alpha>0
$$

With F satisfying a kind of planar negative feedback condition and some appropriate hypotheses, we state an existence theorem for nonconstant periodic solutions.

Neglecting the boundedness condition on $F$, we show the existence of a sequence of values of $\alpha, \alpha_{0}<\alpha_{1}<\ldots$, where a Hopf bifurcation occurs.
\end{abstract}




\section{Conteúdo}

Introdução

1 Definições e Fatos Básicos 1

2 As Bifurcações de Hopf 11

3 Soluções Periódicas $\quad 19$

4 Exemplos $\quad 38$

Referências $\quad 44$ 


\section{Introdução}

Neste trabalho estudamos o problema da existência de soluções periódicas não constantes da equação diferencial com retardamento

$$
x^{\prime}(t)=-x(t)+\alpha F(x(t-1)),
$$

onde $x=\operatorname{col}\left(x_{1}, x_{2}\right) \in \mathbf{R}^{2}, F=\operatorname{col}\left(F_{1}, F_{2}\right)$ é uma função de classe $C^{3}$ de $\mathbf{R}^{2}$ em $\mathbf{R}^{2}$ e $\alpha>0$ é um parâmetro.

No capítulo um apresentamos definições e fatos básicos necessários para o desenvolvimento do trabalho.

A localização das raízes características da equação linearizada de $(E)$, em torno de $(0,0)$, é fundamental para o comportamento assintótico das soluções. Por esse motivo, não podemos deixar de fazer o estudo da equação característica que está contido no capítulo dois. Ainda nesse capítulo provamos, para uma certa classe de funções $F$, a existência de uma infinidade de valores positivos do parâmetro $\alpha$ onde ocorrem bifurcações de Hopf.

No capítulo três, acrescentando uma hipótese de limitação para $F$, fazemos uma aplicação de um teorema de ponto fixo através do qual obtemos a existência de soluções periódicas não constantes da equação $(E)$, com período $T>4$, para $\alpha$ maior que um certo $\alpha_{0}$.

No capítulo quatro apresentamos exemplos mostrando que a classe de funções $F$ para a qual uma equação do tipo $(E)$ admite soluções periódicas não constantes com período $T>4, \alpha$ maior que um certo $\alpha_{0}$, é bastante geral.

Este trabalho visa uma apresentação de detalhes de [8] e a construção de alguns exemplos que consideramos interessantes. A linha de demonstração do Lema 3.7 tem inspiração em [1]. 


\section{Capítulo 1}

\section{Definições e Fatos Básicos}

O interesse central deste trabalho está contido na teoria das equações diferenciais diferença. Como estas são um caso especial das Equações Diferenciais Funcionais Retardadas, formularemos os pré-requisitos no contexto mais geral destas últimas, de acordo com a teoria desenvolvida por Jack Hale em [4].

Consideremos $r \geq 0$ um número real dado, $\mathbf{R}^{n}$ o espaço vetorial $\mathbf{n}$-dimensional com a norma euclideana $|\cdot|, C\left([a, b], \mathbf{R}^{n}\right)$ o espaço de Banach das funções contínuas do intervalo $[a, b]$ tomando valores em $\mathbf{R}^{n}$, munido da norma do supremo.

Sejam $[a, b]=[-r, 0]$ e $C=C\left([-r, 0], \mathbf{R}^{n}\right)$, onde a norma de um elemento $\phi \in C$ é dada por

$$
|\phi|=\sup _{-r \leq \theta \leq 0}|\phi(\theta)|
$$

Definição 1.1 Sejam $\sigma \in \mathbf{R}, A \geq 0$ e $x \in C\left([\sigma-r, \sigma+A], \mathbf{R}^{n}\right)$. Para cada $t \in[\sigma, \sigma+A]$, definimos a função $x_{t} \in C$ por

$$
x_{t}(\theta)=x(t+\theta)
$$

onde $-r \leq \theta \leq 0$.

Definição 1.2 Sejam $D$ um subconjunto de $\mathbf{R} \times C, f: D \rightarrow \mathbf{R}^{n}$ uma função, $e$ “" representando a derivada à direita. A equação 


$$
x^{\prime}(t)=f\left(t, x_{t}\right)
$$

é dita uma equação diferencial funcional retardada sobre $D$ e será denotada por $\operatorname{EDFR}(f)$.

Observação: Seja $F: \mathbf{R}^{n} \times \mathbf{R}^{n} \rightarrow \mathbf{R}^{n}$ uma aplicação contínua. Para $r>0$, uma equação do tipo

$$
x^{\prime}(t)=F(x(t), x(t-r))
$$

é um exemplo de equação diferencial-diferença, objeto de estudo deste trabalho. Se definirmos $f: \mathbf{R} \times C \rightarrow \mathbf{R}^{n}$ por $f(t, \phi)=F(\phi(0), \phi(-r))$, vê-se imediatamente que (1.2) é um caso particular de (1.1), com $D=\mathbf{R} \times C$.

Definição 1.3 Se existem $\sigma \in \mathbf{R}$ e $A>0$ tal que $x \in C\left([\sigma-r, \sigma+A), \mathbf{R}^{n}\right),\left(t, x_{t}\right) \in D$ e $x(t)$ satisfaz a equação (1.1) para $t \in[\sigma, \sigma+A)$, a função $x$ é dita solução da equação (1.1).

Definição 1.4 Dados $\sigma \in \mathbf{R}, \phi \in C$, dizemos que $x(\sigma, \phi, f)$ é uma solução da equação (1.1) com valor inicial $\phi$ em $\sigma$ se:

1. existe $A>0$ tal que $x(\sigma, \phi, f)$ é uma solução da equação (1.1) sobre $[\sigma-r, \sigma+A)$

2. $x_{\sigma}(\sigma, \phi, f)=\phi$

Definição 1.5 A equação (1.1) é dita linear se $f(t, \phi)=L(t, \phi)+h(t)$, onde $L(t, \phi)$ é linear em $\phi$; linear homogênea se $h \equiv 0$ e linear não homogênea se $h \not \equiv 0$.

Definição 1.6 A equação (1.1) é dita autônoma se $f(t, \phi)=g(\phi)$ onde $g$ não depende de $t$.

Lema 1.1. Se $\sigma \in \mathbf{R}, \phi \in C$ são dados e $f(t, \phi)$ é contínua, então encontrar uma solução da equação (1.1) por $(\sigma, \phi)$ é equivalente a resolver a equação integral 


$$
\begin{aligned}
x_{\sigma} & =\phi \\
x(t) & =\phi(0)+\int_{\sigma}^{t} f\left(s, x_{s}\right) d s, \quad t \geq \sigma .
\end{aligned}
$$

Definição 1.7 Sejam $X, Y$ espaços de Banach e $f: A \subset X \rightarrow Y$. A função $f$ é completamente contínua se é contínua e leva cada limitado de $A$ em um conjunto relativamente compacto de $Y$.

Lema 1.2 (Teorema do ponto fixo de Schauder). Se U é um subconjunto limitado, fechado e convexo de um espaço de Banach $X$ e, $T: U \rightarrow U$ é completamente contínua, entâo $T$ tem um ponto fixo em $U$.

Se $V$ é um subconjunto de $\mathbf{R} \times C$, então $C\left(V, \mathbf{R}^{n}\right)$ é a classe de todas funções $f: V \rightarrow \mathbf{R}^{n}$ que são contínuas e $C^{\circ}\left(V, \mathbf{R}^{n}\right) \subset C\left(V, \mathbf{R}^{n}\right)$ é o subconjunto das funções contínuas e limitadas de $V$ em $\mathbf{R}^{n}$. Com a norma

$$
|f|_{V}=\sup _{(t, \phi) \in V}|f(t, \phi)|
$$

o espaço $C^{\circ}\left(V, \mathbf{R}^{n}\right)$ é um espaço de Banach.

Teorema 1.1 (Existência). Sejam $\Omega$ um subconjunto aberto em $\mathbf{R} \times C$ e $f^{\circ} \in$ $C\left(\Omega, \mathbf{R}^{n}\right)$. Se $(\sigma, \phi) \in \Omega$, então existe uma solução da EDFR $\left(f^{\circ}\right)$ passando por $(\sigma, \phi)$.

Mais geralmente, se $W \subseteq \Omega$ é compacto e $f^{\circ} \in C\left(\Omega, \mathbf{R}^{n}\right)$ é dada, então existe uma vizinhança $V \subseteq \Omega$ de $W$ tal que $f^{\circ} \in C^{\circ}\left(V, \mathbf{R}^{n}\right)$, existe uma vizinhança $U \subseteq$ $C^{\circ}\left(V, \mathbf{R}^{n}\right)$ de $f^{\circ}$ e um $\alpha>0$ tal que, para qualquer $(\sigma, \phi) \in W, f \in U$, existe uma solução $x(\sigma, \phi, f)$ da $\operatorname{EDFR}(f)$ por $(\sigma, \phi)$ definida em $[\sigma-r, \sigma+\alpha]$.

Teorema 1.2 (Dependência contínua). Suponhamos que $\Omega \subseteq \mathbf{R} \times C$ é aberto, $\left(\sigma^{o}, \phi^{o}\right) \in \Omega, f^{0} \in C\left(\Omega, \mathbf{R}^{n}\right)$ e $x^{o}$ é uma soluçâo da $\operatorname{EDFR}\left(f^{\circ}\right)$ por $\left(\sigma^{\circ}, \phi^{0}\right)$ que existe e é única em $\left[\sigma^{\circ}-r, b\right]$. Sejam $W^{\circ} \subseteq \Omega$ o conjunto compacto definido por

$$
W^{\circ}=\left\{\left(t, x_{t}^{o}\right) \mid t \in\left[\sigma^{\circ}, b\right]\right\}
$$

$e, V^{\circ}$ uma vizinhança de $W^{\circ}$ na qual $f^{\circ}$ é limitada. Se $\left(\sigma^{k}, \phi^{k}, f^{k}\right), k=1,2, \ldots$ satisfaz $\sigma^{k}, \rightarrow \sigma^{\circ}, \phi^{k} \rightarrow \phi^{\circ} e\left|f^{k}-f^{0}\right|_{V^{\circ} \rightarrow 0}$ quando $k \rightarrow \infty$, então existe um $k^{o}$ tal que para $k \geq k_{o}$ cada solução $x^{k}=x^{k}\left(\sigma^{k}, \phi^{k}, f^{k}\right)$ por $\left(\sigma^{k}, \phi^{k}\right) d a \operatorname{EDFR}\left(f^{k}\right)$ 
existe em $\left[\sigma^{k}-r, b\right]$ e $x^{k} \rightarrow x^{\circ}$ uniformemente em $\left[\sigma^{\circ}-r, b\right]$. Como nem todas as $x^{k}$ estão necessariamente definidas em $\left[\sigma^{\circ}-r, b\right]$, por $x^{k} \rightarrow x^{\circ}$ uniformemente sobre $\left[\sigma^{\circ}-r, b\right]$, entendemos que, para qualquer $\epsilon>0$, existe $k_{1}(\epsilon)$ tal que $x^{k}(t), k \geq k_{1}(\epsilon)$, está definida sobre $\left[\sigma^{\circ}-r+\epsilon, b\right]$ e $x^{k} \rightarrow x^{\circ}$ uniformemente sobre $\left[\sigma^{\circ}-r+\epsilon, b\right]$.

Teorema 1.3 (Unicidade). Suponhamos que $\Omega$ é um conjunto aberto em $\mathbf{R} \times C, f$ : $\Omega \rightarrow \mathbf{R}^{n}$ é contínua e $f(t, \phi)$ é Lipschitziana em $\phi$ em cada conjunto compacto em $\Omega$. Se $(\sigma, \phi) \in \Omega$, então a solução da equação $(1.1)$ por $(\sigma, \phi)$ é única.

Definição 1.8 Suponhamos que $f$ na equação (1.1) seja contínua. Se $x$ é uma solução da equação (1.1) sobre um intervalo $[\sigma-r, a), a>\sigma$, dizemos que $\tilde{x}$ é um prolongamento de $x$ se existe $b>a$ tal que $\tilde{x}$ está definida em $[\sigma-r, b)$, coincide com $x$ em $[\sigma-r, a)$ e $\tilde{x}$ satisfaz a equação $(1.1)$ em $[\sigma, b)$.

Definição 1.9 Uma solução $x$ da equação (1.1) é maximal se não existe um prolongamento de $x$. Neste caso, seu intervalo de existência $[\sigma-r, a)$ é chamado intervalo maximal de existência à direita.

Teorema 1.4 (Escape de compactos). Sejam $\Omega$ um conjunto aberto em $\mathbf{R} \times C e$ $f \in C\left(\Omega, \mathbf{R}^{n}\right)$. Se $x$ é uma solução maximal da equação (1.1) sobre $[\sigma-r, b)$, então, para qualquer conjunto compacto $W$ em $\Omega$, existe um $t_{W}$ tal que $\left(t, x_{t}\right) \notin W$ para $t_{W} \leq t<b$.

Teorema 1.5 (Escape de limitados e fechados). Sejam $\Omega$ um conjunto aberto em $\mathbf{R} \times C$ e $f: \Omega \rightarrow \mathbf{R}^{n}$ completamente contínua, isto é, $f$ é contínua e leva conjuntos fechados e limitados de $\Omega$ em conjuntos limitados de $\mathbf{R}^{n}$. Se $x$ é uma solução maximal da equação (1.1) em $[\sigma-r, b)$, então para todo conjunto fechado e limitado $U \subset \Omega$, existe $t_{U} \in \mathbf{R}$ tal que $\left(t, x_{t}\right) \notin U$ para $t \in\left[t_{U}, b\right)$.

Observação: Os fatos apresentados até aqui pertencem a teoria geral das EDFR. No entanto, não os utilizaremos em toda generalidade neste trabalho. $\mathrm{Na}$ verdade, pode-se tirar vantagens das particularidades das equações estudadas a seguir para obter informações mais completas e de forma mais simples. Descrevemos abaixo uma situação que ilustra o que estamos afirmando.

Se $F: \mathbf{R}^{n} \rightarrow \mathbf{R}^{n}$ é uma função contínua pode-se demonstrar diretamente que o problema de valor inicial 


$$
\begin{aligned}
x^{\prime}(t) & =-x(t)+F(x(t-r)), \\
\left.x\right|_{[-r, 0]} & =\phi
\end{aligned}
$$

tem uma única solução $x:[-r, \infty) \rightarrow \mathbf{R}^{n}$.

De fato, essa equação é equivalente à

$$
\begin{aligned}
& \left(e^{t} x(t)\right)^{\prime}=e^{t} F(x(t-r)) \\
& \left.x\right|_{[-r, 0]}=\phi
\end{aligned}
$$

Chamando de $x_{1}(t)$ a solução $x(t)$ para $t \in[0, r],(1.5)$ nos leva a

$$
x_{1}(t)=\phi_{1}(0) e^{-t}+\int_{0}^{t} e^{s-t} F(\phi(s-r)) d s, \quad t \in[0, r]
$$

Do mesmo modo se $x_{2}(t)$ representa a solução $x(t)$ para $t \in[r, 2 r]$, obtemos

$$
x_{2}(t)=x_{1}(r) e^{r-t}+\int_{r}^{t} e^{s-t} F\left(x_{1}(s-r)\right) d s, \quad t \in[r, 2 r]
$$

Procedendo dessa forma sucessivamente obtemos a existência e a unicidade da solução de (1.4) em $[-r, \infty) . \diamond$

Definição 1.10 Consideremos uma familia a um parâmetro de EDFRs da forma

$$
x^{\prime}(t)=F\left(\alpha, x_{t}\right)
$$

onde $F(\alpha, \phi)$ tem primeira e segunda derivadas contínuas em $\alpha, \phi$, para $\alpha \in \mathbf{R}$, $\phi \in C$ e $F(\alpha, 0)=0$ para todo $\alpha$. Definamos $L: \mathbf{R} \times C \rightarrow \mathbf{R}^{n}$ por

$$
L(\alpha) \psi=F_{\phi}(\alpha, 0) \psi,
$$

onde $F_{\phi}(\alpha, 0)$ é a derivada de $F(\alpha, \phi)$ com respeito a $\phi$ em $\phi=0$.

Consideremos as seguintes hipóteses:

$\left(H_{1}\right) \quad$ A EDFR $(\mathrm{L}(0))$ linear tem uma raiz característica simples imaginária pura $\lambda_{0}=i \nu_{0} \neq 0$ e toda raiz característica $\lambda_{j} \neq \lambda_{0}, \bar{\lambda}_{0}$ satisfaz $\lambda_{j} \neq m \lambda_{0}$ para todo 
inteiro $\mathrm{m}$. Uma vez que $L(\alpha)$ é continuamente diferenciável em $\alpha$, de acordo com [4, Lema 2.2, seção 7.2], existe $\alpha_{0}>0$ e uma raiz característica simples $\lambda(\alpha)$ da equação linear $\operatorname{EDFR}(\mathrm{L}(\alpha))$ a qual tem uma derivada contínua $\lambda^{\prime}(\alpha)$ em $\alpha$ para $|\alpha|<\alpha_{0}$.

$\left(H_{2}\right) \quad \Re\left(\lambda^{\prime}\right)(0) \neq 0$, onde a linha representa a derivada de $\lambda$ em relação a $\alpha$.

O Teorema enunciado a seguir é conhecido como Teorema de Bifurcação de Hopf.

Teorema 1.6. Se $F(\alpha, \phi)$ tem primeira e segunda derivadas contínuas com relação a $\alpha$ e $\phi, F(\alpha, 0)=0$ para todo $\alpha$, e as hipóteses $\left(H_{1}\right)$ e $\left(H_{2}\right)$ estão satisfeitas, então existem constantes $a_{0}>0, \alpha_{0}>0, \delta_{0}>0$, funções continuamente diferenciáveis $\alpha(a) \in \mathbf{R}, \omega(a) \in \mathbf{R}$ e $x^{*}(a) \omega\left(\right.$ a)-periódica, para $|a|<a_{0}$, tal que $x^{*}(a)$ é uma solução da equação (1.6). Além disso, para $|\alpha|<\alpha_{0},\left|\omega-\left(\frac{2 \pi}{\nu_{0}}\right)\right|<\delta_{0}$, as soluçóes $x^{*}$ são as únicas soluções $\omega$-periódicas da equação (1.6) com $\left|x_{t}\right|<\delta_{0}$, exceto por uma translą̧ão de fase.

Seja $K$ um espaço métrico compacto e $M$ um espaço métrico. Consideremos $C(K, M)$ o conjunto das aplicaçôes contínuas de $K \mathrm{em} M$ com a topologia dada por

$$
d(f, g)=\max _{x \in K} d(f(x), g(x))
$$

onde $f, g \in C(K, M)$.

Definição 1.11 Um conjunto $E \subset C(K, M)$ é equicontínuo se para todo $\epsilon>0$ existe $\delta>0, \delta=\delta(\epsilon)$ tal que se $d(x, y)<\delta$, então $d(f(x), f(y))<\varepsilon, \forall f \in E$.

Daremos a seguir uma versão do teorema de Ascoli útil aos nossos propósitos. Teorema 1.7 (Ascoli-Arzela). Sejam $K$ um espaço métrico compacto e $E \subset C\left(K, \mathbf{R}^{m}\right)$. Se

1. existe $N \geq 0$ tal que $|f(x)| \leq N, \forall x \in K, \forall f \in E$,

2. E é equicontínuo,

entâo $E$ é relativamente compacto.

$\mathrm{O}$ conceito de ejetividade, dado a seguir, é atribuído a Browder [2]. 
Definição 1.12 Sejam $X$ um espaço de Banach, $U$ um subconjunto de $X$ e $x$ um ponto de $U$. Dada uma aplicação $A: U \backslash\{x\} \rightarrow X$, o ponto $x \in U$ é dito um ponto ejetivo de $A$ se existe uma vizinhança aberta $G \subseteq X$ de $x$, tal que para cada $y \in G \cap U, y \neq x$, existe um inteiro $m=m(y)$ tal que $A^{m} y \notin G \cap U$.

Definição 1.13 Dado $M>0$, definimos $S_{M}=\{x \in X|| x \mid=M\}$ e $B_{M}=\{x \in$ $X|| x \mid<M\}$.

Observamos que $S_{M}=\partial B_{M}$.

Teorema 1.8. Se $K$ é um subconjunto fechado, limitado, convexo, de dimensão infinita de um espaço de Banach $X, A: K \backslash\left\{x_{0}\right\} \rightarrow K$ é completamente contínua, $e x_{0} \in K$ é um ponto ejetivo de $A$, então existe um ponto fixo de $A$ em $K \backslash\left\{x_{0}\right\}$. Se $K$ é de dimensão finita e $x_{0}$ é um ponto extremo de $K$, então é válida a mesma conclusão.

O Teorema abaixo é atribuído a R. Nussbaum [6].

Teorema 1.9. Sejam $X$ um espaço de Banach, $K$ um subcojunto convexo e fechado de $X$ e $A: K \backslash\{0\} \rightarrow K$ uma aplicação completamente contínua, tal que $0 \in K$ é um ponto ejetivo de $A$. Suponhamos que exista um $M>0$ tal que $A x=\lambda x$ implique $\lambda<1$ para todo $x \in K \cap S_{M}$. Então $A$ tem um ponto fixo em $K \cap B_{M} \backslash\{0\}$ se $K$ tem dimensão infinita ou 0 é um ponto extremo de $K$.

Consideremos as equações diferenciais retardadas

$$
\begin{gathered}
x^{\prime}(t)=L x_{t}+f\left(x_{t}\right) \\
y^{\prime}(t)=L y_{t}
\end{gathered}
$$

onde $L: C \rightarrow \mathbf{R}^{2}$ é uma aplicação linear contínua, $f: C \rightarrow \mathbf{R}^{2}$ é completamente contínua com sua primeira derivada contínua, $f(0)=0$ e $D f(0)=0$. A equação característica da equação (1.8) é dada por

$$
\operatorname{det} \Delta(\lambda)=0
$$

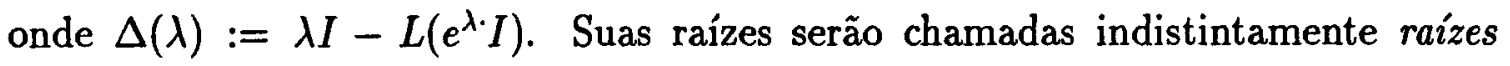


características ou autovalores.

Para cada autovalor $\lambda$, o espaço de fase $\mathrm{C}$ é decomposto como soma direta, $C=P_{\lambda} \oplus Q_{\lambda}$, onde $P_{\lambda}$ e $Q_{\lambda}$ são invariantes sob o operador solução da equação (1.8), $T(t), t \geq 0$ dado por $T(t) \phi=y_{t}(\cdot ; \phi), \phi \in C$. Seja $\pi_{\lambda}$ o operador projeção com imagem $P_{\lambda}$ definido por essa decomposição.

Seja $\Phi=\left(\phi_{1 \lambda}, \ldots, \phi_{d \lambda}\right)$ uma base do subespaço $P_{\lambda}$ e consideremos a aplicação linear $\phi \rightarrow b$, onde $b=b(\phi)$ é um d-vetor definido por $\pi_{\lambda} \phi=\Phi b$. Escolhemos para $b$ a norma euclideana.

Podemos então enunciar o Lema abaixo cuja demonstração é encontrada em [3, Lema 3.1] ou [4,capítulo 10, Lema 1.1].

Lema 1.3. Existe uma forma quadrática definida positiva $V(\phi)=b^{T} B b$, com a seguinte propriedade:

$\forall p>0, \exists \delta_{0}>0$ tal que $\forall \delta, \quad 0<\delta \leq \delta_{0}$, se $\phi$ satisfaz $\|\phi\| \leq \delta$ e $V(\phi) \geq p^{2} \delta^{2}$, então

$$
V^{\prime}(\phi):=\liminf _{t \rightarrow 0_{+}} \frac{1}{t}\left[V\left(x_{t}(. ; \phi)\right)-V(\phi)\right]>\frac{1}{2} V(\phi)>0
$$

Lema 1.4. Suponhamos que as seguintes condições estejam satisfeitas:

(i) Existe um autovalor $\lambda$ da equação (1.8) $\operatorname{com} \Re(\lambda)>0$.

(ii) Existe um subconjunto convexo fechado $K$ de $C, 0 \in K$, e uma função contínua $\tau: K \backslash\{0\} \rightarrow[\rho, \infty), \rho>0$, tal que a aplicação $A: K \rightarrow C$ dada por

$$
\begin{aligned}
& A \phi=x_{\tau(\phi)}(\cdot ; \phi), \quad \phi \in K \backslash\{0\} \\
& A 0=0
\end{aligned}
$$

é completamente contínua e $A K \subset K$.

(iii) Se $0<a<M$, para algum $M \in \mathbf{R}$,

$$
\inf \left\{\left\|\pi_{\lambda} x_{t}\right\| \mid x_{t}=x_{t}(\cdot ; \phi), \phi \in K,\|\phi\|=a, 0 \leq t \leq \tau(\phi)\right\}>0 \text {. }
$$


(iv) Dado $G \subset C$ aberto, $0 \in G$, existe uma vizinhança $V$ de 0 tal que $x_{t}(\cdot ; \phi) \in G$, se $\phi \in V \cap K, \phi \neq 0, e 0 \leq t \leq \tau(\phi)$.

Então, $O$ é um ponto ejetivo de $A$.

Prova: Sejam $\phi \in K$ e $x_{t}=x_{t}(\cdot ; \phi)$ solução não nula de (1.7).

Chamando de $b(t)$ a coordenada de $\frac{x_{t}}{\left\|x_{t}\right\|}$ em $P_{\lambda}$ e considerando a função $V(\phi)$ do Lema 1.3, segue que:

$$
V\left(\frac{x_{t}}{\left\|x_{t}\right\|}\right)=b(t)^{T} B b(t)=|b(t)|^{2} \frac{b(t)^{T}}{|b(t)|} B \frac{b(t)}{|b(t)|} \geq|b(t)|^{2} \beta^{2}
$$

onde $\beta^{2}:=\min \left\{b^{T} B b|| b \mid=1\right\}$.

Então

$$
\left\|\pi_{\lambda}\left(\frac{x_{t}}{\left\|x_{t}\right\|}\right)\right\|^{2} \leq\|\Phi\|^{2}|b(t)|^{2} \leq \frac{\|\Phi\|^{2}}{\beta^{2}} V\left(\frac{x_{t}}{\left\|x_{t}\right\|}\right)=u^{2} \beta^{-2} V\left(\frac{x_{t}}{\left\|x_{t}\right\|}\right)
$$

Segue de (1.10) e da hipótese (iii) que

$$
\nu^{2}:=\inf \left\{V\left(\frac{x_{t}}{\left\|x_{t}\right\|}\right) \mid x_{t}=x_{t}(\cdot ; \phi), \phi \in K,\|\phi\|=a, 0 \leq t \leq \tau(\phi)\right\}>0
$$

Fixemos p, $0<p<\nu$ e seja $\delta_{0}$ de acordo com o lema 1.3.

Então para todo $\delta, 0<\delta \leq \delta_{0}$, se

$$
\|\phi\| \leq \delta, V(\phi) \geq p^{2} \delta^{2} \Rightarrow V^{\prime}(\phi)>\frac{1}{2} V(\phi)>0
$$

Para cada $\delta, 0<\delta \leq \delta_{0}$, definamos

$$
G(\delta):=\left\{\phi \in C \mid\|\phi\|<\delta, V(\phi)<p^{2} \delta^{2}\right\} .
$$

$G(\delta)$ é aberto e $0 \in G(\delta)$. 
Escolhamos $\delta_{1}, 0<\delta_{1}<\delta_{0}$ de modo que segundo a condição (iv) do Lema 1.4,

$$
\phi \in G\left(\delta_{1}\right) \cap K, \phi \neq 0 \Rightarrow x_{t} \in G\left(\delta_{0}\right), \quad 0 \leq t \leq \tau(\phi)
$$

Para $\phi \in G\left(\delta_{1}\right) \cap K, \phi \neq 0$ e $t \in[0, \tau(\phi)]$ fixado arbitrariamente, seja $\epsilon=\left\|x_{t}(. ; \phi)\right\|<\delta_{0}$

\section{Então}

$$
V\left(x_{t}\right)=\left\|x_{t}\right\|^{2} V\left(\frac{x_{t}}{\left\|x_{t}\right\|}\right) \geq \varepsilon^{2} \nu^{2}>p^{2} \varepsilon^{2}
$$

e o lema 1.3 implica que

$$
V^{\prime}\left(x_{t}\right)>\frac{1}{2} V\left(x_{t}\right)>\frac{1}{2} p^{2} \epsilon^{2}>0
$$

isto é, $V\left(x_{t}\right)$ é estritamente crescente em $t, 0 \leq t \leq \tau(\phi)$.

Além disso, $V^{\prime}\left(x_{t}\right)$ fica uniformemente afastada do zero em $[0, \tau(\phi)]$, já que

$$
V^{\prime}\left(x_{t}\right)>\frac{1}{2} V\left(x_{t}\right)>\frac{1}{2} V(\phi) .
$$

Se $A \phi=x_{\tau(\phi)}(. ; \phi)$ ainda permanecer em $G\left(\delta_{1}\right) \cap K$, nós podemos repetir o raciocínio para assegurar que

$$
V^{\prime}\left(x_{t}\right)>\frac{1}{2} V\left(x_{t}\right)>\frac{1}{2} V\left(x_{\tau(\phi))}>\frac{1}{2} V(\phi)>0\right.
$$

para $\tau(\phi) \leq t \leq \tau(\phi)+\tau(A \phi)$ e assim sucessivamente.

Suponhamos por um momento que $A^{n}(\phi) \in G\left(\delta_{1}\right) \cap K, \quad n=1,2 \ldots$

Então $V\left(x_{t}(. ; \phi)\right)$ é estritamente crescente em $t, V(\phi)<p^{2} \delta_{1}^{2}$ e $V^{\prime}\left(x_{t}(. ; \phi)\right)$ fica uniformemente longe do zero para $t \geq 0$.

Sejam $t_{0}$ o primeiro instante $t$ para o qual $V\left(x_{t_{0}}(\cdot ; \phi)\right)=p^{2} \delta_{0}^{2}$ e $n_{0}=n_{0}(\phi)$ o maior inteiro positivo tal que $A^{n_{0}} \phi=x_{T}$ para algum $T \leq t_{0}$.

Então, como $x_{t_{0}} \notin G\left(\delta_{0}\right), t_{0} \leq T+\tau\left(A^{n_{0}} \phi\right)$ pois $p^{2} \delta_{0}^{2}=V\left(x_{t_{0}}\right)$, segue da construção de $\delta_{1}$ que

$$
A^{n_{0}} \phi \notin G\left(\delta_{1}\right) \cap K
$$

o que é uma contradição, e portanto, temos a ejetividade de 0 com respeito à $A$. $\diamond$ 


\section{Capítulo 2}

\section{As Bifurcações de Hopf}

Consideremos a família a um parâmetro de equações diferenciais autônomas não lineares da forma

$$
x^{\prime}(t)=-x(t)+\alpha F(x(t-1))
$$

onde $x=\operatorname{col}\left(x_{1}, x_{2}\right) \in R^{2}, F: R^{2} \rightarrow R^{2}$ de classe $C^{3}, F(0)=0, F^{\prime}(0) \neq 0$ e $\alpha>0$ um parâmetro real. Nosso objetivo neste capítulo, é estabelecer para certos valores do parâmetro $\alpha$, a existência de soluções periódicas não nulas de $(E)$, que emanam da solução nula - as chamadas Bifurcações de Hopf.

Denotando $\frac{\partial F_{1}}{\partial x_{2}}(0)=\delta_{1}$ e $\frac{\partial F_{2}}{\partial x_{1}}(0)=\delta_{2}$, a equação $(E)$ com $F$ munida da hipótese:

$$
x_{2} F_{1}(x)>0, \text { se } x_{2} \neq 0, x_{1} F_{2}(x)<0 \text {, se } x_{1} \neq 0 ; \delta_{1}=-\delta_{2}=1
$$

tem linearização próxima à origem dada por:

$$
x^{\prime}(t)=-x(t)+B(\alpha) x(t-1)
$$

onde

$$
B(\alpha):=\left(\begin{array}{rr}
0 & \alpha \\
-\alpha & 0
\end{array}\right)
$$

Observação: As hipóteses $x_{2} F_{1}(x)>0, x_{2} \neq 0$ e $x_{1} F_{2}(x)<0, x_{1} \neq 0$ implicam $\delta_{1},-\delta_{2} \geq 0$. 
As suposições sobre as derivadas parciais podem ser substituídas por $\delta_{1}, \delta_{2} \neq 0$.

Observemos também que as hipóteses acima forçam que as órbitas da equação $(E)$ espiralem em torno da origem e tornam plausível a existência de soluções periódicas.

Uma condição necessária e suficiente para a existência de uma solução não trivial de $(L)$ da forma $x(t)=e^{\lambda t} u, u \in \mathbf{R}^{2}$, é que $\lambda$ seja raiz da chamada equação característica de $(L)$ :

$$
\operatorname{det} \Delta(\lambda)=0, \Delta(\lambda):=(\lambda+1) I-B(\alpha) e^{-\lambda}
$$

onde I representa a matriz identidade de ordem 2; ou equivalentemente

$$
(\lambda+1)^{2} e^{2 \lambda}=-\alpha^{2}
$$

Fazendo uma mudança de parâmetros $\beta=\alpha e$ e uma translação de $\lambda, \mu=\lambda+1$, a equação (2.2) torna-se

$$
\mu^{2} e^{2 \mu}=-\beta^{2}
$$

Observemos que $\mu=0$ é a única raiz real de (2.3) e ocorre somente quando $\beta=0$. Além disso, $\mu$ é uma raiz de (2.3) se e somente se, seu complexo conjugado $\bar{\mu}$ também o é; podemos, portanto, restringir nosso estudo ao semiplano superior $\Im(\mu)>0$.

Lema 2.1. As raízes imaginárias puras de (2.3) são $\mu= \pm k \pi i$, e elas ocorrem apenas para $\beta=k \pi, k=1,2, \ldots$.

Prova: Se $\mu=a+b i,(2.3)$ é equivalente ao sistema:

$$
\left\{\begin{array}{l}
e^{2 a}\left[\left(a^{2}-b^{2}\right) \cos 2 b-2 a b \text { sen } 2 b\right]=-\beta^{2} \\
\left(a^{2}-b^{2}\right) \text { sen } 2 b+2 a b \cos 2 b=0
\end{array}\right.
$$

Supondo $\mu=b i, b>0$, o sistema (2.4) nos dá sen $2 b=0, \cos 2 b>0$ e, portanto, $b=k \pi$ e $\beta=k \pi, \quad k=1,2, \ldots \diamond$ 
Lema 2.2. Não existe raizes de (2.3) sobre as retas $a+b i \quad \mid \quad b=\left(k+\frac{1}{2}\right) \pi$, $k=0,1,2, \ldots$.

Prova: Se $\mu=a+\left(k+\frac{1}{2}\right) \pi i, \quad k=0,1,2, \ldots$, o sistema (2.4) torna-se:

$$
\left\{\begin{array}{cc}
e^{2 a} & {\left[a^{2}-\left(k+\frac{1}{2}\right)^{2} \pi^{2}\right]=\beta^{2}} \\
& a(2 k+1) \pi=0
\end{array}\right.
$$

o qual é incompatível. $\diamond$

Segue do Lema 2.2 que toda raiz $\mu$ de (2.3) deve pertencer a alguma das faixas:

$$
S_{k}:=\left\{\mu=a+b i, b>0,\left(k-\frac{1}{2}\right) \pi<b<\left(k+\frac{1}{2}\right) \pi\right\}, \quad k=0,1,2, \ldots .
$$

Lema 2.3. Se $\mu=a+b i \in S_{0}$ é uma raiz de (2.3), entâo $a>0$.

Prova: Segue da equação (2.3) que $\mu e^{\mu}$ é um número imaginário puro. Portanto, como $\Re\left(\mu e^{\mu}\right)=e^{a}[a \cos b-b \operatorname{sen} b]=0, \operatorname{com} \cos b>0$ e $b$ sen $b>0$, concluímos que $a$ deve ser positivo. $\diamond$

Lema 2.4. Cada faixa $S_{k}, k=0,1,2, \ldots$ contém precisamente uma raiz de (2.3), que é simples.

Prova: Faremos a prova para a faixa $S_{1}$. Pequenas adaptações levam aos demais casos.

Seja $\mu=a+b i \in S_{1}$. A equação (2.3) implica $\Re\left(\mu e^{\mu}\right)=e^{a}(a \cos b-b \operatorname{sen} b)=0 \mathrm{e}$, como $\cos b<0$, segue que $a \operatorname{sen} b=\frac{b \operatorname{sen}^{2} b}{\cos b} \leq 0$. Logo, $\Im\left(\mu e^{\mu}\right)=e^{a}(a \operatorname{sen} b+b \cos b)$ é negativo, de modo que na faixa com a qual estamos tratando, (2.3) é equivalente à:

$$
\mu e^{\mu}=-i \beta
$$

e a equação (2.5) pode ser reescrita como:

$$
\left\{\begin{array}{l}
b \tan b=a \\
e^{a}(a \operatorname{sen} b+b \cos b)=-\beta
\end{array}\right.
$$


Substituindo a primeira equação de (2.6) na 2a. equação, obtemos

$$
\beta=\Lambda(b):=\frac{-b e^{b \tan b}}{\cos b}
$$

de modo que:

$$
\begin{gathered}
\frac{d \Lambda}{d b}=-e^{b \tan b}\left[\sec b+b \sec b \tan b+b \sec b\left(\tan b+b \sec ^{2} b\right)\right] \\
=-e^{b \tan b}\left(1+2 b \tan b+b^{2} \sec ^{2} b\right) \sec b \\
=-e^{b \tan b}\left[(1+b \tan b)^{2}+b^{2}\right] \sec b>0
\end{gathered}
$$

e além disso,

$$
\Lambda\left(\frac{\pi}{2}+\right)=0, \Lambda\left(\frac{3 \pi}{2}-\right)=+\infty
$$

As propriedades de $\Lambda$ implicam que (2.7) define $b$ univocamente como uma função de $\beta, 0<\beta<+\infty$. Através da primeira equação de (2.6), também obtemos $a$ e, portanto $\mu \in S_{1}$, como função de $\beta$. Além disso, $\mu$ é diferenciável com respeito à $\beta$.

Seja $\mu_{0}$ uma raiz de (2.3). Uma vez que $\mu_{0} \neq-1$ obtemos

$$
\frac{d}{d \mu}\left(\mu^{2} e^{2 \mu}+\beta^{2}\right)_{\mu=\mu_{0}}=2 \mu_{0} e^{2 \mu_{0}}\left(1+\mu_{0}\right) \neq 0
$$

portanto, $\mu_{0}$ é simples. $\diamond$

Observação: Da prova do Lema 2.4 decorre que:

1. Se $\mu$ permanece no semiplano superior $\Im(\mu)>0$, em cada faixa fixada $S_{k}$ a raiz de (2.3) é uma função diferenciável de $\beta>0$ e, em virtude de (2.8) temos que $\frac{d b}{d \beta}>0$.

2. Como $|\tan b|<b \sec ^{2} b$, em virtude da 1a. equação de (2.6)

$$
\frac{d a}{d \beta}=\left(\tan b+b \sec ^{2} b\right) \frac{d b}{d \beta}>0
$$


Lema 2.5. Se $\mu_{k}=a_{k}+b_{k} i \in S_{k}, \quad k=0,1,2, \ldots$ são raizes de (2.3), então suas partes reais satisfazem $a_{0}>a_{1}>\ldots \rightarrow-\infty$.

Prova: Se $\mu^{2} e^{2 \mu}=-\beta^{2}$ e $\mu=a+b i$, então

$$
\left(a^{2}+b^{2}\right) e^{2 a}=\beta^{2}
$$

Para $\beta=\pi$ a equação (2.10) define $b>0$ como função de $a, a \in(-\infty, 0]$ e tal função é decrescente, já que

$$
\frac{d b}{d a}=-\frac{1}{b}\left[\pi^{2} e^{-2 a}+a\right]<0, \text { para } a \leq 0 .
$$

Ainda, de acordo com os Lemas 2.1 e 2.3 temos que $a_{0}>a_{1}=0>a_{k}, \quad k=$ $2,3, \ldots$.

Assim $a_{0}>a_{1}=0>\ldots>a_{k} \ldots$, com $a_{k}+b_{k} i=\mu_{k} \in S_{k}$, para $k \geq 0$.

Suponhamos por um momento que tal ordenação seja falsa para algum valor do parâmetro $\beta>0$.

Como $\Re(\mu)$ é uma função contínua de $\beta$ em cada faixa $S_{k}$, existe algum $\beta=\bar{\beta}$ para o qual (2.3) tem raízes $\mu_{k}$ e $\mu_{l}$ tal que, $a_{k}=a_{l}=a, \operatorname{com} k>l$.

Mas então, pela equação (2.10):

$$
\left(a^{2}+b_{k}^{2}\right) e^{2 a}=\left(a^{2}+b_{l}^{2}\right) e^{2 a}
$$

o que implica que $b_{k}=b_{l}$, uma contradição.

Assim, $a_{0}>a_{1}>\ldots$ Se $a=a_{k}, b=b_{k}$ em (2.10), podemos ver que $a_{k} \rightarrow-\infty$, quando $k \rightarrow \infty$. $\diamond$

Relembrando que as raizes $\mu$ da equação (2.3) se distribuem simetricamente com relação ao eixo real, os Lemas 2.1 - 5 fornecem uma descrição completa do lugar geométrico dessas raízes. Quando $\beta$ varia de 0 à $+\infty$, as raízes de (2.3) descrevem as curvas $a=b \tan b$ em $S_{k}, k=0,1, \ldots$. A figura 2.1(a) representa uma situação particular para um valor fixado de $\beta$. $O$ modo como as raízes $\mu$ estão distribuídas sobre as curvas $a=b \tan b$ é vinculado a ordenação das partes reais estabelecida no Lema 2.5 . 


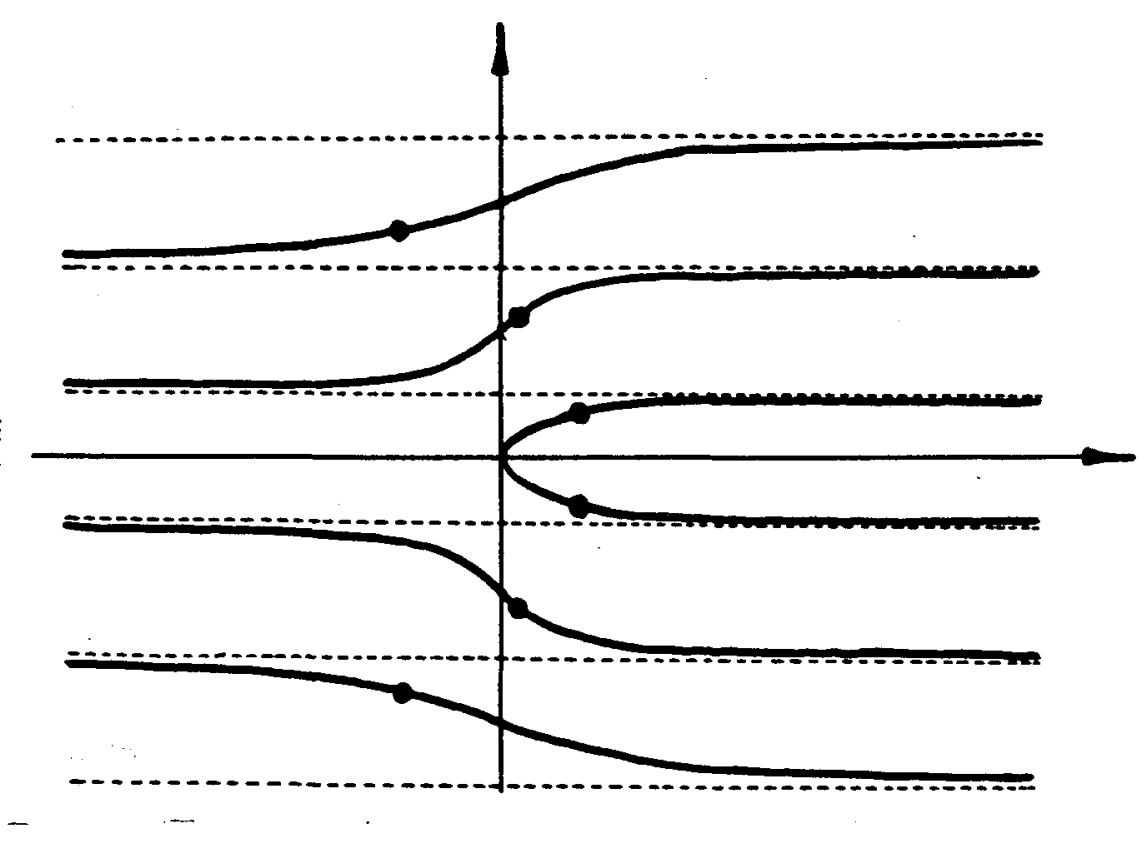

Figura 2.1(a). Localização das raízes de (2.3)

Quando $\alpha$ atinge um certo valor $\alpha_{0}$, da esquerda para direita, um par de autovalores conjugados $\lambda_{0}=\lambda\left(\alpha_{0}\right), \bar{\lambda}_{0}$, cruzam o eixo imaginário pela primeira vez. Como os autovalores $\lambda$ de $(L)$ são as translações $\lambda=\mu-1$ das raízes $\mu$ de (2.3), $\alpha_{0}$ corresponde ao valor $\beta_{0}$ de $\beta$ que produz $\mu \in S_{0}$ da forma $\mu=1+b i$ de modo que:

$$
\begin{aligned}
& \Re\left(\mu e^{\mu}\right)=e[\cos b-b \operatorname{sen} b]=0, \\
& \Im\left(\mu e^{\mu}\right)=e[\operatorname{sen} b+b \cos b]=\beta_{0} .
\end{aligned}
$$

Alguns cálculos elementares leva-nos à $e\left(1+b^{2}\right)$ sen $b=\beta_{0}$ e $\alpha_{0}=\left(1+b^{2}\right)$ sen $b$, onde $b$ é dado por $b \tan b=1,0<b<\frac{\pi}{2}$.

De acordo com observação subsequente ao lema 2.4 segue que $\frac{d}{d \beta}(\Re(\mu))>0$. Levando em conta que $\alpha$ é um múltiplo positivo de $\beta$, podemos dizer que a parte real de $\lambda$ cruza o eixo imaginário com velocidade positiva.

A localização dos autovalores $\lambda$ de $(L)$ é representada na Figura 2.1 (b) exatamente como uma translação da Figura 2.1 (a). 


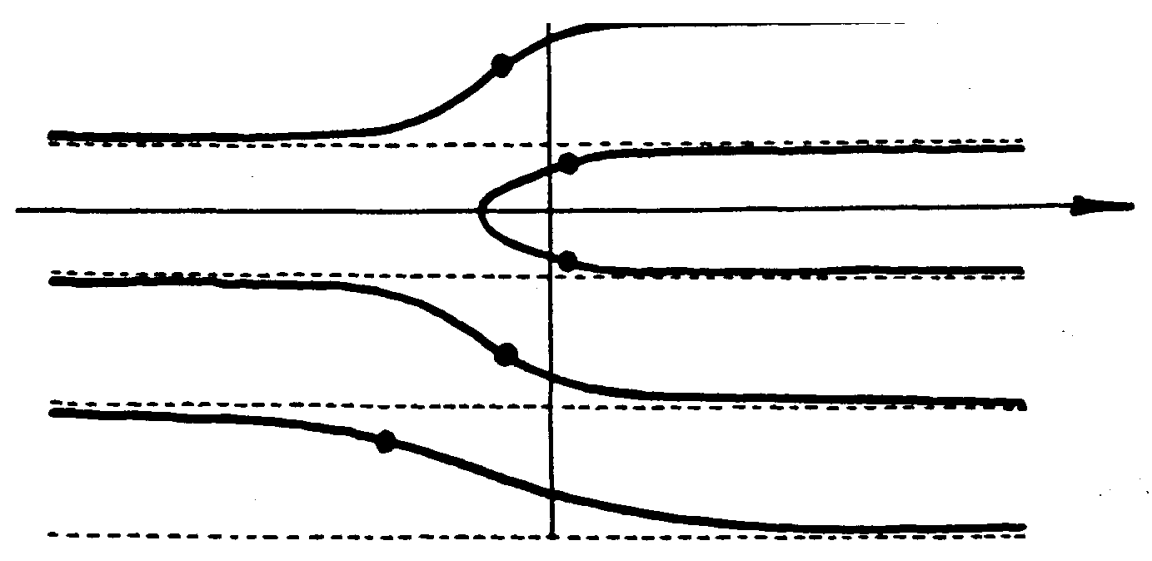

Figura 2.1 (b). Localização dos autovalores de (2.2).

Teorema 2.1. Se (H.1) é satisfeita, então existe uma sequência $\alpha_{0}<\alpha_{1}<$ $\ldots \rightarrow \infty$ tal que a equação (E) tem uma bifurcação de Hopf em $\alpha=\alpha_{k}, k=0,1, \ldots$

Prova: A sequência $\alpha_{0}<\alpha_{1}<\ldots$ corresponde aos valores de $\alpha$ para os quais um par de autovalores conjugados cruza o eixo imaginário. Sua monotonicidade segue da ordenação das partes reais dos $\lambda_{k}^{\prime} s$, dada no Lema 2.5 , e da monotonicidade da parte real de $\lambda$ com respeito a $\alpha$.

Umá vez que o espectro de uma equação diferencial linear com retardamento tem infinitos autovalores à esquerda do eixo imaginário, temos que $\alpha_{k} \rightarrow \infty$, quando $k \rightarrow \infty$.

O teorema 2.1 é agora uma consequência imediata das seguintes afirmações as quais seguem diretamente dos lemas e observações anteriores:

(i) Os autovalores imaginários puros $\lambda_{k}=\lambda_{k}\left(\alpha_{k}\right) \neq 0, k=0,1, \ldots$ são simples e, para qualquer $k$ fixado, os autovalores $\lambda_{j} \neq \lambda_{k}, \overline{\lambda_{k}}$ satisfazem $\lambda_{j} \neq m \lambda_{k}$, para todo inteiro $m$.

(ii) $\frac{d}{d \alpha}\left[\Re\left(\lambda_{k}(\alpha)\right)\right]_{\alpha=\alpha_{k}} \neq 0 . \diamond$ 
Teorema 2.2. Se (H.1) é satisfeita, a equação

$$
x^{\prime}(t)=\beta F(x(t-1)), \quad \beta>0
$$

tem uma bifurcação de Hopf em cada valor $\beta=k \pi, k=1,2, \ldots$.

Prova: Como a equação característica da parte linear da equação (2.11) é precisamente a equação (2.3), segue que a prova do Teorema 2.2 nada mais é do que uma repetição dos argumentos anteriores. $\diamond$ 


\section{Capítulo 3}

\section{Soluções Periódicas}

Neste capítulo provamos a existência de soluções periódicas não triviais de $(E)$, para uma certa classe de aplicações $F$, para $\alpha>$ algum $\alpha_{0}$ positivo. Isso é feito mostrando que um certo operador de retorno definido pelo fluxo num cone do espaço de fase $C\left([-1,0], \mathbf{R}^{2}\right)$, tem ponto fixo não trivial.

A maior dificuldade desse procedimento se deve ao fato de que a origem é um ponto fixo, correspondente à solução periódica trivial, conhecido "a priori". Essa dificuldade é contornada usando um teorema de ponto fixo, Teorema 1.9, que depende do conceito de ejetividade e garante a existência de pontos fixos não nulos.

O objeto de interesse central a partir de agora é a família $(E)$.

Dada $\phi \in C=C\left([-1,0], \mathbf{R}^{2}\right)$, seja $x(. ; \phi)$ a solução de $(E)$ definida em $[-1, \infty)$ tal que $\left.x\right|_{[-1,0]}=\phi$. É conveniente termos as equações $(E)$ também escritas na forma:

$$
\begin{aligned}
& x_{1}^{\prime}(t)=-x_{1}(t)+\alpha F_{1}(x(t-1)) \\
& x_{2}^{\prime}(t)=-x_{2}(t)+\alpha F_{2}(x(t-1))
\end{aligned}
$$

Além da hipótese (H.1) assumida no capítulo 2, consideremos também neste capítulo a hipótese

(H.2) Existe $M>0$ tal que $|F(x)| \leq M, \forall x \in \mathbf{R}^{2}$. 
O subconjunto fechado e convexo de C,

$K=\left\{\phi \in C \mid e^{\theta} \phi_{j}(\theta)\right.$ não decrescentes em $\left.[-1,0], j=1,2 ; \phi_{1}(-1)=0, \phi_{2}(-1) \geq 0\right\}$

é de importância decisiva neste capítulo. Embora não tiremos vantagem disto, salientamos que $K$ satisfaz as condições da definição de cone, isto é, $K$ é um subconjunto fechado e convexo do espaço de Banach $C, \lambda K \subset K$ se $\lambda \geq 0$ e $K \cap(-K)=\{0\}$.

Teorema 3.1. Seja $\alpha_{0}=\left(1+b^{2}\right)$ sen $b$, com $b$ univocamente definido por $b \tan b=1$ em $\left[0, \frac{\pi}{2}\right]$. Se as hipóteses (H.1) e (H.2) estão satisfeitas, então para todo $\alpha>\alpha_{0}$, a equação (E) tem uma solução periódica não trivial $x(. ; \phi), \phi \in K$, com período $T>4$.

A prova deste teorema é baseada na existência de pontos fixos não triviais de um operador $A: K \rightarrow K$, obtido acompanhando-se o fluxo da equação $(E)$, isto é,

$$
A \phi=\left\{\begin{array}{l}
x_{\tau}(. ; \phi), \phi \in K \backslash\{0\}, \text { para algum } \tau=\tau(\phi)>0 \\
0, \phi=0
\end{array}\right.
$$

Essa estratégia depende do Teorema 1.9 e é a evolução de uma idéia devida à Jones [5].

Provamos, a seguir, alguns lemas que permitem definir tal operador de retorno e conduzem à ejetividade da origem.

Lema 3.1. Se $F$ é uma função limitada, então qualquer solução $x(. ; \phi), \phi \in C, d a$ equação (E) é limitada para $t \geq-1$.

Prova: Da equação $(E)$, segue que

$$
x(t) \cdot x^{\prime}(t)=-|x(t)|^{2}+\alpha x(t) \cdot F(x(t-1))
$$

onde "." representa o produto escalar do $R^{2}$.

Assim,

$$
\begin{gathered}
x(t) \cdot x^{\prime}(t) \leq-|x(t)|^{2}+\alpha|x(t)||F(x(t-1))| \\
=-|x(t)|[|x(t)|-\alpha|F(x(t-1))|] \leq-|x(t)|[|x(t)|-\alpha M], t \geq 0
\end{gathered}
$$


onde $\mathrm{M}$ é dado na hipótese ( $H .2)$.

Seja $N>\alpha M$. Se para algum $t_{0} \geq 0,\left|x\left(t_{0}\right)\right| \geq N,(3.2)$ implica que

$$
x\left(t_{0}\right) . x^{\prime}\left(t_{0}\right) \leq-N[N-\alpha M]<0 .
$$

Portanto $\forall N>\alpha M, \exists \tau \geq 0$ tal que $x(t) \in B_{N}$ para $t \geq \tau$, o que completa a prova. $\diamond$

Da prova do Lema 3.1 segue que se $x(t ; \phi)$ é solução periódica de $(E)$ então $|x(t ; \phi)| \leq \alpha M, \forall t \in \mathbf{R}$.

Daqui em diante indicamos por $Q_{1}$ e $Q_{2}$ o primeiro e o quarto quadrantes do plano $x_{1} x_{2}$, isto é,

$$
\begin{aligned}
& Q_{1}:=\left\{\left(x_{1}, x_{2}\right) \in R^{2} \mid x_{1} \geq 0, x_{2} \geq 0\right\}, \\
& Q_{2}:=\left\{\left(x_{1}, x_{2}\right) \in R^{2} \mid x_{1} \geq 0, x_{2} \leq 0\right\} .
\end{aligned}
$$

Lema 3.2. Suponhamos que as hipóteses (H.1) e (H.2) estão satisfeitas. Então, existe uma função contínua $t_{1}: K \backslash\{0\} \rightarrow[0, \infty)$ tal que $x_{1}\left(t_{1}(\phi) ; \phi\right)>0, x(t ; \phi) \in$ $Q_{1}$ se $0 \leq t \leq t_{1}(\phi)$ e $x(t ; \phi) \in$ int $\left(Q_{2}\right)$ se $t_{1}(\phi)<t<t_{1}(\phi)+\delta$ para qualquer $\delta>0$ suficientemente pequeno.

Prova: Suponhamos que para alguma $\phi \in K \backslash\{0\}$, não exista $t_{1} \geq 0$ tal que $x(t ; \phi)=x(t) \in Q_{1}$ para $0 \leq t \leq t_{1}, x_{1}\left(t_{1}\right)>0, x_{2}\left(t_{1}\right)=0$.

De $\phi=\left(\phi_{1}, \phi_{2}\right) \in K \backslash\{0\}$, segue que $\left(\phi_{1}(0), \phi_{2}(0)\right) \neq(0,0)$.

Consideremos $\phi_{2}(0)>0$. Observemos que não há perda de generalidade em considerar também que $\phi_{1}(0)>0$, se não, tomamos a condição inicial $\psi=x_{\tau}(. ; \phi)$ para um conveniente $\tau>0$.

A equação $(E a)$ e a hipótese ( $H .1)$ implicam $x_{1}(t) \geq \phi_{1}(0) e^{-t}>0$ enquanto $x(t-1)$ permanecer no primeiro quadrante $Q_{1}$; portanto $x(t ; \phi)$ não sai de $Q_{1}$ pelo eixo $x_{2}$.

Se assumirmos que $x(t) \in Q_{1},-1 \leq t<\infty$, a equação $(E b)$ e a hipótese $(H .1)$ implicam que $x_{2}^{\prime}(t) \leq-x_{2}(t) \leq 0$, ou seja, $x_{2}(t)$ é não crescente e, portanto, devemos ter 


$$
\lim _{t \rightarrow \infty} x_{2}(t)=\eta \geq 0
$$

Se $\eta>0, \exists \bar{t}>0$ tal que $x_{2}(t)>\frac{\eta}{2}$ para $t \geq \bar{t}$ e então, por $(E b)$,

$$
x_{2}(t) \leq x_{2}(\bar{t})-\frac{\eta}{2}(t-\bar{t}), t \geq \bar{t}
$$

o que implica que $x_{2}(t)$ se torna negativa num tempo finito e isto contradiz o fato que $x(t) \in Q_{1},-1 \leq t<\infty$. Assim

$$
\lim _{t \rightarrow \infty} x_{2}(t)=0
$$

Afirmamos que

$$
\lim _{t \rightarrow \infty} x_{1}(t)=0
$$

Mostremos primeiramente que não podemos ter $x_{1}(t) \rightarrow \xi>0$, quando $t \rightarrow \infty$. Como $F_{1}(\xi, 0)=0$, se $\lim _{t \rightarrow \infty} x_{1}(t)=\xi>0$, segue da equação $(E a)$ que $\lim _{t \rightarrow \infty} x_{1}^{\prime}(t)=-\xi<0$, assim $x_{1}(t)$ fica negativa a partir de um tempo finito, $\mathrm{e}$ isso contradiz o fato de que $x(t) \in Q_{1},-1 \leq t<\infty$.

Pelo Lema $3.1,\left|x_{1}(t)\right|<N$ para algum $N>0$.

Suponhamos, por um momento, que (3.5) não seja verdade. Então, existe $\xi \in R$ tal que $0 \leq \lim _{t \rightarrow \infty}$ inf $x_{1}(t)<\xi<\lim _{t \rightarrow \infty} \sup x_{1}(t) \leq N<\infty$ e uma sequência $\left(t_{n}\right) \rightarrow \infty, n=1,2, \ldots, \operatorname{com} x_{1}\left(t_{n}\right)=\xi, x_{1}^{\prime}\left(t_{n}\right) \geq 0$, o que é uma contradição já que por $(E a)$,

$$
x_{1}^{\prime}\left(t_{n}\right)=-\xi+\alpha F_{1}\left(x_{1}\left(t_{n}-1\right), x_{2}\left(t_{n}-1\right)\right) \rightarrow-\xi \text { quando } t_{n} \rightarrow \infty .
$$

Assim vale (3.5) e portanto, sempre que $x(t ; \phi)$ permanecer em $Q_{1}$ para $t \geq 0$, as equações (3.4) e (3.5) dão que

$$
\lim _{t \rightarrow \infty} x(t ; \phi)=0
$$


A hipótese $(H .1)$ implica que $F_{2}\left(0, x_{2}\right)=0$, de modo que a expansão de Taylor de $F_{2}(x)$ próximo à origem é

$$
F_{2}(x)=-x_{1}+x_{1}\left[a x_{1}+b x_{2}+O\left(|x|^{2}\right)\right]
$$

quando $x \rightarrow 0$, e isto implica que dado $0<\delta<1$, existe uma vizinhança $V$ do zero no $\mathbf{R}^{2}$ tal que se $x \in V, x_{1}>0$,

$$
F_{2}(x)<(-1+\delta) x_{1}
$$

Por (3.6), não há perda de generalidade em assumir $x(t ; \phi) \in V \cap Q_{1}$, para $-1 \leq t<\infty, x_{1}(-1)>0$, caso contrário tomamos como condição inicial $\psi=x_{\tau}(. ; \phi)$ para $\tau>0$ suficientemente grande.

Como $(E b)$ é equivalente a

$$
\begin{aligned}
& x_{2}(t)=\phi_{2}(t), \quad t \in[-1,0] \\
& x_{2}(t)=x_{2}(0) e^{-t}+\alpha \int_{0}^{t} e^{s-t} F_{2}(x(s-1)) d s, \quad t \geq 0
\end{aligned}
$$

segue por (3.7) que

$$
\begin{gathered}
x_{2}(n+1)=x_{2}(0) e^{-n-1}+\alpha \int_{0}^{n+1} e^{s-n-1} F_{2}(x(s-1)) d s< \\
<x_{2}(0) e^{-n-1}-\alpha(1-\delta) \int_{0}^{n+1} e^{s-n-1} x_{1}(s-1) d s= \\
=x_{2}(0) e^{-n-1}-\alpha(1-\delta) e^{-n} \int_{0}^{n+1} e^{s-1} x_{1}(s-1) d s .
\end{gathered}
$$

Já que $e^{s-1} x_{1}(s-1)$ é uma função não decrescente de $s$ para $s \geq 0$,

$$
\begin{aligned}
x_{2}(n+1) & <x_{2}(0) e^{-n-1}-\alpha(1-\delta) e^{-n} \int_{0}^{n+1} e^{-1} x_{1}(-1) d s \\
= & e^{-n-1}\left[x_{2}(0)-(n+1) \alpha(1-\delta) x_{1}(-1)\right]
\end{aligned}
$$

o que implica que $x_{2}(t)$ se torna negativa num tempo finito, uma contradição. 
Portanto $\exists t_{1} \geq 0$ tal que

$$
x(t ; \phi) \in Q_{1} \text { para } 0 \leq t \leq t_{1}, x_{1}\left(t_{1} ; \phi\right)>0, x_{2}\left(t_{1} ; \phi\right)=0
$$

Como $\phi_{2}(0)>0$ então $t_{1}>0$ e $x_{1}\left(t_{1}-1\right)>0$ tomando, se necessário, uma condição inicial $\psi=x_{\tau}(. ; \phi)$ para um $\tau>0$ conveniente.

Assim, segue da equação $(E b)$ que $x(t ; \phi)$ atravessa transversalmente o semi-eixo horizontal no instante $t=t_{1}$ e definimos $t_{1}(\phi)=t_{1}$.

Se $\phi_{2}(0)=0$ é fácil ver que existe $0 \leq \tau<1$ tal que $x_{2}(t) \equiv 0, x_{1}(t)>0$ para $-1<t \leq \tau$. Neste caso, a solução $x(t ; \phi)$ deixa $Q_{1}$ no instante $t=\tau$, tangencialmente, e $t_{1}(\phi)=\tau$.

A continuidade de $t_{1}$ segue do Teorema da Continuidade em relação às Condições Iniciais, Teorema 1.2.

Lema 3.3. Seja $G \subset C$ aberto, $0 \in G$. Existe uma vizinhança $V$ de 0 em $C$ tal que $\forall \phi \in V \cap K, \phi \neq 0$, tem-se $x_{t}(. ; \phi) \in G$ para $0 \leq t \leq t_{1}(\phi)+1$.

Prova: É suficiente provar que dado $G \subset \mathbf{R}^{2}$ aberto, $0 \in G$, existe uma vizinhança $V$ de 0 em $C$ tal que se $\phi \in V \cap K, \phi \neq 0$ então $x(t ; \phi) \in G$ para $-1 \leq t \leq t_{1}(\phi)+1$.

Admitindo que vale tal afirmação para $t \in\left[-1, t_{1}(\phi)\right]$, como $F$ é contínua, $F(0)=0$, segue das equações $(E a),(E b)$ e hipótese $(H .1)$ que

$$
\begin{aligned}
& \left(e^{t} x_{1}(t)\right)^{\prime} \leq \alpha e^{t} \epsilon \\
& \left(e^{t} x_{2}(t)\right)^{\prime} \geq-\alpha e^{t} \epsilon,
\end{aligned}
$$

com $t \in\left[t_{1}(\phi), t_{1}(\phi)+1\right]$, para qualquer $\epsilon>0$, desde que $|x(t ; \phi)|$ seja suficientemente pequeno em $\left[-1, t_{1}(\phi)\right]$.

Essas desigualdades implicam que $0 \leq x_{1}(t ; \phi) \leq \alpha \epsilon$ e $-\alpha \epsilon \leq x_{2}(t ; \phi) \leq 0$ para $t \in\left[t_{1}(\phi), t_{1}(\phi)+1\right]$.

Suponhamos então que a afirmação não seja verdadeira para $t \in\left[-1, t_{1}(\phi)\right]$. Então existe retângulo aberto 


$$
R=\left\{\left(x_{1}, x_{2}\right) \mid-\eta_{1}<x_{1}<l,-\eta_{2}<x_{2}<m\right\} \ni(0,0)
$$

tal que para alguma sequência $\left(\phi_{n}\right), \phi_{n} \in K \backslash\{0\}, \phi_{n} \rightarrow 0$ quando $n \rightarrow \infty, \exists\left(t_{n}\right)$, $-1 \leq t_{n} \leq t_{1}\left(\phi_{n}\right)$ com $x\left(t_{n} ; \phi_{n}\right) \in \partial R, n=1,2, \ldots$.

Sejam $x_{n}(t)=x\left(t ; \phi_{n}\right), x_{1 n}(t) \geq 0$ e $x_{2 n}(t) \geq 0$ enquanto $x_{n}(t)$ pertencer ao primeiro quadrante $Q_{1}$. Além disso, $x_{2 n}(t)$ é decrescente se $\phi_{2 n}(0)>0$ ou $x_{2 n}(t) \equiv 0$ se $\phi_{2 n}(0)=0$ para $0 \leq t \leq t_{1}\left(\phi_{n}\right)$.

Assim, $x_{n}(t) \in[0, l] \times\left[0, q_{n}\right],-1 \leq t \leq t_{n}$, onde $q_{n}=\left\|\phi_{n}\right\| \rightarrow 0$ quando $n \rightarrow \infty$.

Consequentemente, $x_{1}\left(t_{n} ; \phi_{n}\right)=l$ para $n=1,2, \ldots$.

A continuidade de $F_{1}$ e $F_{1}\left(x_{1}, 0\right) \equiv 0$ implicam que $\lim _{x_{2} \rightarrow 0} F_{1}\left(x_{1}, x_{2}\right)=0$ uniformemente em $x_{1}, 0 \leq x_{1} \leq l$.

Assim podemos escrever, enquanto $x_{n}(t) \in[0, \eta] \times\left[0, q_{n}\right]$,

$$
\begin{gathered}
x_{1 n}(t)=\phi_{1 n}(0) e^{-t}+\alpha e^{-t} \int_{0}^{t} e^{s} F_{1}\left(x_{n}(s-1)\right) d s \leq \\
\leq q_{n} e^{-t}+\alpha e^{-t} M_{n}\left[e^{t}-1\right] \\
\leq q_{n} e^{-t}+\alpha M_{n}\left[1-e^{-t}\right]
\end{gathered}
$$

onde $M_{n}=\sup \left\{F_{1}(x) \mid x \in[0, \eta] \times\left[0, q_{n}\right]\right\}$.

Segue disto que $x_{1}\left(t_{n} ; \phi_{n}\right)<q_{n}+\alpha M_{n}<l$ para $n$ suficientemente grande, o que contradiz a escolha de $\phi_{n}$. $\diamond$

Dada $\phi \in K \backslash\{0\}$, seja $\psi=x_{t_{1}(\phi)+1}(. ; \phi)$ e observemos que, para $-1 \leq \theta \leq 0$,

$$
\left(e^{\theta} \psi(\theta)\right)^{\prime}=\alpha e^{\theta} F\left(x\left(t_{1}(\phi)+\theta ; \phi\right)\right)
$$

Uma vez que $x\left(t_{1}(\phi)+\theta ; \phi\right) \in Q_{1}$, para $-1 \leq \theta \leq 0$, a relação (3.8) e a hipótese (H.1) implicam que $e^{\theta} \psi_{1}(\theta)$ é não decrescente e $e^{\theta} \psi_{2}(\theta)$ é não crescente. 
Assim, se $R$ é a rotação

$$
R=\left(\begin{array}{rr}
0 & 1 \\
-1 & 0
\end{array}\right)
$$

e $R K:=\{R \phi \mid \phi \in K\}$, essas propriedades de $e^{\theta} \psi(\theta)$ implicam que $\psi \in R K$.

Podemos então, definir a aplicação

$$
\begin{aligned}
A_{1}: K & \longrightarrow R K \\
A_{1} \phi & =x_{t_{1}(\phi)+1}(. ; \phi), \quad \phi \neq 0 \\
A_{1} 0 & =0
\end{aligned}
$$

Lema 3.4. A aplicação $A_{1}: K \rightarrow R K$ é completamente contínua.

Prova: A continuidade de $t_{1}$ e da solução de $(E)$ em relação às condições iniciais garantem a continuidade de $A_{1}$ em $K \backslash\{0\}$. Em $\phi=0$ a continuidade segue do Lema 3.3.

Seja $\bar{B}_{\delta}$ uma bola fechada em $C$ e $V=K \cap \bar{B}_{\delta}$. Então

$$
A_{1}(V)=\left\{x_{t_{1}(\phi)+1}(. ; \phi) \mid \phi \in V \backslash\{0\}\right\} \cup\{0\}
$$

Os mesmos argumentos da prova do Lema 3.1 implicam que $A_{1}(V)$ é limitado por $\max \{\alpha M ; \delta\}$.

A equicontinuidade de $A_{1}(V)$ segue da limitação uniforme das derivadas que, por sua vez, é uma consequência da continuidade do campo vetorial $H: \mathbf{R}^{2} \times \mathbf{R}^{2} \rightarrow \mathbf{R}^{2}$ definido por $H(u, v)=-u+\alpha F(v)$. $\diamond$

Destacamos que as hipóteses assumidas até agora possuem uma simetria rotacional, de modo que podemos repetir todos os passos da construção de $A_{1}$, através dos Lemas 3.2, 3.3 e 3.4 com adaptações óbvias, para definir as funções contínuas $\phi \rightarrow t_{j}(\phi)$ de $R^{j-1}(K \backslash\{0\})$ em $[0, \infty), j=2,3,4$ e dessas funções obter as funções completamente continuas

$$
A_{j}: R^{j-1} K \longrightarrow R^{j} K, j=2,3,4 .
$$

Agora, definimos a aplicação

$$
\begin{aligned}
A: K & \longrightarrow R^{4} K=K \\
A(\phi) & =\left(A_{4} \circ A_{3} \circ A_{2} \circ A_{1}\right)(\phi) .
\end{aligned}
$$


Se para cada $\phi \in K \backslash\{0\}$ chamarmos de $\tau(\phi)$ o instante para o qual $A(\phi)=$ $x_{\tau(\phi)}(. ; \phi)$, segue imediatamente da construção de $A$ que

$$
\tau(\phi)=t_{1}(\phi)+t_{2}\left(A_{1} \phi\right)+t_{3}\left(A_{2} A_{1} \phi\right)+t_{4}\left(A_{3} A_{2} A_{1} \phi\right)+4
$$

O último termo, "4", aparece nesta expressão em virtude do efeito do retardamento a cada vez que a solução $x(t ; \phi)$ cruza um eixo coordenado.

Portanto, de acordo com o Lema 3.2 e da expressão (3.10) segue a continuidade da função

$$
\tau: K \backslash\{0\} \longrightarrow[4, \infty)
$$

A figura 3.1 ilustra uma situação típica do processo descrito.

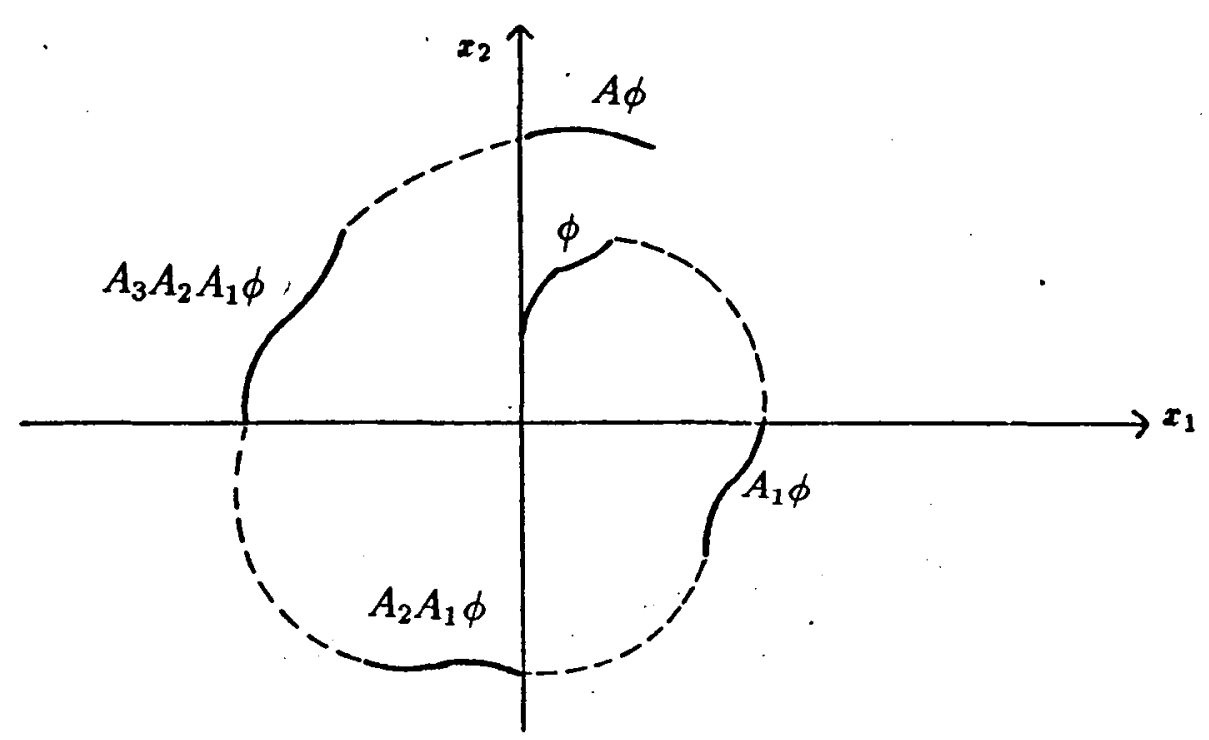

Figura 3.1

O lema seguinte é consequência imediata do Lema 3.3 e das simetrias das nossas hipóteses.

Lema 3.5. Seja $G \subset C$ aberto, $0 \in G$. Existe uma vizinhança $V$ de 0 em $C$ tal que se $\phi \in V \cap K, \phi \neq 0$ então $x_{t}(. ; \phi) \in G$ para $0 \leq t \leq \tau(\phi)$. 
Lema 3.6. Existe um conjunto fechado convexo $K \subset C, 0 \in K$, e uma função contínua $\tau: K \backslash\{0\} \rightarrow[4, \infty)$ tal que a função

$$
\begin{aligned}
A: K & \rightarrow C \\
A(\phi) & =x_{\tau(\phi)}(. ; \phi), \phi \in K \backslash\{0\} \\
A(0) & =0,
\end{aligned}
$$

é completamente contínua e $A K \subset K$.

Prova: Consequência imediata do Lema 3.4 e observações após a sua prova. $\diamond$

Relembramos agora a decomposição do espaço de fase como uma soma direta, $C=P_{\lambda} \oplus Q_{\lambda}$, associada a um autovalor $\lambda$ da equação característica (2.2). Uma apresentação completa deste assunto é encontrada em [4, cap7], aqui apenas recordamos alguns fatos que são relevantes para o nosso problema específico.

Assumimos $\alpha>\alpha_{0}:=\left(1+b^{2}\right)$ sen $b$, onde $b$ é univocamente definido em $\left[0, \frac{\pi}{2}\right]$ pela relação $b \tan b=1$. Portanto, segundo discussão após a prova do Lema 2.5, podemos escolher um autovalor $\lambda$ de $(L)$, com $\Re(\lambda)>0$ que é simples. Além disso, tomamos $\lambda$ tal que $0<\Im(\lambda)<\frac{\pi}{2}$.

A equação $(L)$, linearizada de $(E)$ em torno da origem, é um caso particular da equação

$$
y^{\prime}(t)=L\left(y_{t}\right)
$$

onde $L: C \rightarrow \mathbf{R}^{2}, L(\phi)=-\phi(0)+B(\alpha) \phi(-1)$.

Seja $\eta(\theta), \theta \in[-1,0]$, matriz de ordem 2 definida por

$$
\eta(\theta)= \begin{cases}0, & \theta=-1 \\ B(\alpha), & -1<\theta<0 \\ -I+B(\alpha), & \theta=0\end{cases}
$$

Então para qualquer $\phi \in C$,

$$
L(\phi)=\int_{-1}^{0}[d \eta(\theta)] \phi(\theta) .
$$

Seja $G$ o gerador infinitesimal do semigrupo $\{T(t), t \geq 0\}$ definido pelas soluções de (3.11),

$$
T(t) \phi=y_{t}(. ; \phi), \phi \in C, t \geq 0 .
$$


Então, $\lambda$ é um autovalor simples de $G$ e o subespaço $P_{\lambda}$ é precisamente o autoespaço generalizado 1- dimensional associado a $\lambda, M_{\lambda}(G)$.

Se $G^{*}$ é o operador adjunto formal de $G, \lambda$ é também autovalor de $G^{*}$ e seu autoespaço generalizado $M_{\lambda}\left(G^{*}\right)$ é 1- dimensional.

Seja $C^{*}=C\left([0,1], \mathbf{R}^{2^{*}}\right)$, onde $\mathbf{R}^{2^{*}}$ é o plano dos vetores linhas. A forma bilinear de $C^{*} \times C$ dada por

$$
\left\langle\psi, \phi>:=\psi(0) . \phi(0)-\int_{-1}^{0} \int_{0}^{\theta} \psi(\xi-\theta)[d \eta(\theta)] \phi(\xi) d \xi\right.
$$

$\psi \in C^{*}, \phi \in C$, "." significando produto escalar do $\mathbf{R}^{2}$, aparece de maneira natural na decomposição de $C$.

Se $u$ é uma solução não trivial de $\Delta(\lambda) u=0, \operatorname{com} \Delta(\lambda):=(\lambda+1) I-B(\alpha) e^{-\lambda}$ dado em (2.1), e o vetor linha $v$ é uma solução não trivial de $v \Delta(\lambda)=0$, então as funções $\rho(t):=e^{\lambda t} u, t \in[-1,0]$ e $\sigma(s):=v e^{-\lambda s}, s \in[0,1]$, geram $M_{\lambda}(G)$ e $M_{\lambda}\left(G^{*}\right)$ respectivamente.

Além disso, relembramos que se $\phi \in C=P_{\lambda} \oplus Q_{\lambda}$ é decomposto como $\phi=\phi^{P_{\lambda}}+\phi^{Q_{\lambda}}$ então sua componente $\phi^{P_{\lambda}}$ em $P_{\lambda}$ é dada por

$$
\phi^{P_{\lambda}}=\langle\sigma, \phi>\rho
$$

Lema 3.7. Para qualquer constante $a, 0<a<\alpha M$,

$$
\inf \left\{\left\|\pi_{\lambda}\left(x_{t}\right)\right\| \mid x_{t}=x_{t}(. ; \phi), \phi \in K,\|\phi\|=a, 0 \leq t \leq \tau(\phi)\right\}>0 .
$$

A prova deste lema segue dos cinco lemas subsequentes.

Observemos que, se $\phi \in K$,

$$
\|\phi\| \geq d \Rightarrow|\phi(0)| \geq d e^{-1}
$$

como consequência de $e^{t}|\phi(t)|$ ser não decrescente.

Os três próximos lemas descrevem um certo comportamento das soluções $x(. ; \phi)$ de $(E)$, dando uma estimativa inferior positiva para $x_{1}\left(t_{1}(\phi) ; \phi\right)\left(t_{1}\right.$ definido no Lema 3.2) uniforme em $\phi \in K,\|\phi\| \geq d$. 
Se $k$ e $\sigma$ são suficientemente pequenos, a figura 3.2 fornece uma visão geométrica útil no entendimento de alguns passos das provas.

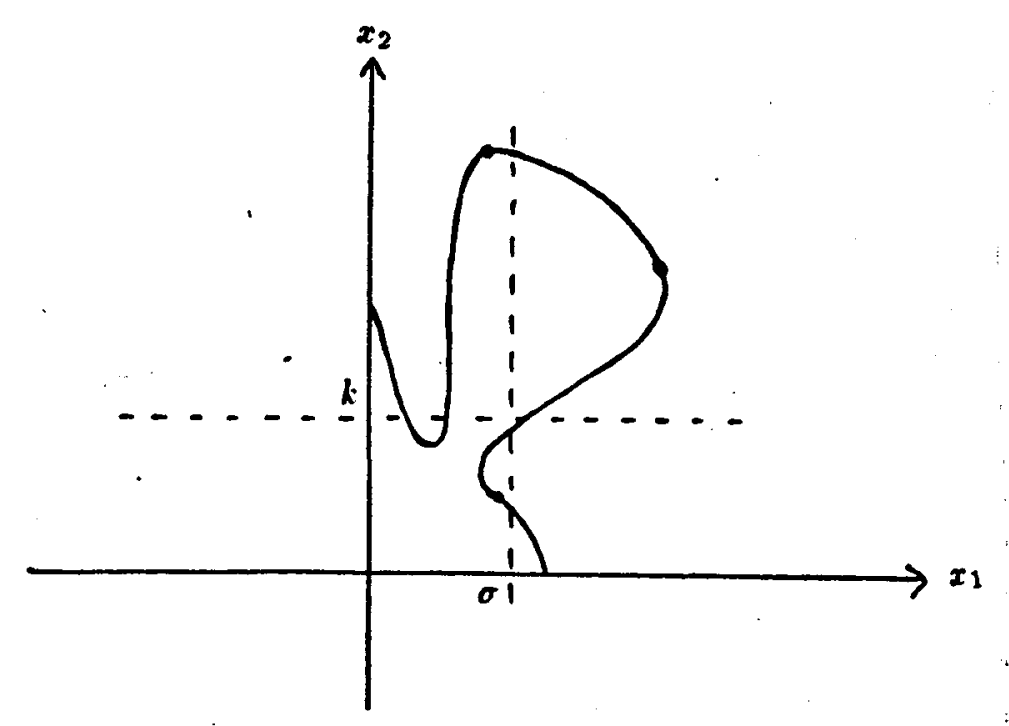

Figura 3.2

Lema 3.8. Seja $d>0$. Então existem $k>0, \sigma>0$ tais que $\forall \phi \in K,\|\phi\| \geq d$, $\phi_{1}(0) \leq \sigma$, existe $\tau_{1}=\tau_{1}(\phi)>0$ satisfazendo $x_{1}\left(\tau_{1} ; \phi\right)>\sigma$ e $x_{2}(t ; \phi) \geq k$ para $t \in\left[0, \tau_{1}\right]$.

Prova: Da hipótese (H.1) segue que a expansão de Taylor de $F_{2}(x)$ perto da origem $\dot{\mathbf{e}}$

$$
F_{2}(x)=-x_{1}+x_{1}\left[a x_{1}+b x_{2}+\mathrm{O}\left(|x|^{2}\right)\right] \text { quando } x \rightarrow 0
$$

Assim, dado $0<\eta<1$, existe vizinhança $V$ de 0 em $\mathbf{R}^{2}$ tal que se $x \in V, x_{1}>0$

$$
F_{2}(x)<(-1+\eta) x_{1}
$$


Seja $\xi$ tão pequeno de modo que $R=\left\{\left(x_{1}, x_{2}\right) \mid-\xi<x_{1}<\xi,-\xi<x_{2}<\xi\right\}$ esteja contido em V.

Definimos:

$$
k:=\min \left\{\frac{d}{4 e^{3}}, \xi\right\}
$$

Como $x_{2} F_{1}(x)>0, x_{2} \neq 0$, então

$$
\exists \sigma^{\prime}>0 \mid\left(x_{1}, x_{2}\right) \in Q_{1}, x_{2} \geq k \Rightarrow F_{1}\left(x_{1}, x_{2}\right) \geq \max \left\{\sigma^{\prime}, \frac{\sigma^{\prime}}{\alpha}\right\}
$$

Como $x_{1} F_{2}(x)<0, x_{1} \neq 0$, da continuidade de $F_{2}(x)$ segue que

$$
\begin{gathered}
\forall \varepsilon>0, \varepsilon<\frac{d}{4 \alpha e^{3}}, \exists \delta=\delta(\varepsilon)>0 \mid\left(x_{1}, x_{2}\right) \in Q_{1}, x_{1} \leq \delta(\varepsilon) \\
\Rightarrow-\varepsilon \leq F_{2}\left(x_{1}, x_{2}\right)<0
\end{gathered}
$$

Seja:

$$
\sigma:=\min \left\{\frac{\delta(\varepsilon)}{e}, \frac{\sigma^{\prime}}{2}, k\right\}
$$

Segue de (3.19), (3.20) e da equação $(E b)$,

$$
x_{2}(t ; \phi) \geq\left[\phi_{2}(0)+\alpha \varepsilon\right] e^{-t}-\alpha \varepsilon, \quad t \in[0,1] .
$$

Além disso $\phi_{2}(0) \geq \frac{d}{2 e}$ pois $\phi_{1}(0) \leq \sigma$ e $\|\phi\| \geq d$.

Assim:

$$
x_{2}(t ; \phi) \geq\left[\frac{d}{2 e}+\alpha \varepsilon\right] e^{-t}-\alpha \varepsilon, \quad t \in[0,1] .
$$

A função $E(t):=\left[\frac{d}{2 e}+\alpha \varepsilon\right] e^{-t}-\alpha \varepsilon$ é uma função decrescente em $[0,1]$ e $E(1)>\frac{d}{4 e^{3}} \geq k$.

Portanto

$$
x_{2}(t ; \phi)>k, \quad t \in[0,1] .
$$

O Lema 3.2 garante a existência de um primeiro instante $\hat{t}>1$ para o qual $x_{2}(\hat{t} ; \phi)=k$ e $x_{2}(t ; \phi)>k$ para $0 \leq t<\hat{t}$. 
Suponhamos por um momento que $x_{1}(t) \leq \sigma$ para todo $t \in[0, \hat{t}]$. Então $x_{1}(t-1) \leq \sigma e \leq \delta(\varepsilon), t \in[0, \hat{t}]$, e portanto $-\varepsilon \leq F_{2}\left(x_{1}, x_{2}\right)<0$.

Repetindo o argumento anterior segue que

$$
x_{2}(t ; \phi) \geq E(t), \quad t \in[0, \hat{t}]
$$

Como $E(2)>\frac{d}{4 e^{3}} \geq k$, temos que $\hat{t}>2$.

Por $(E a)$ e (3.18) segue agora, para $t \in[1,2]$,

$$
x_{1}^{\prime}(t) \geq-\sigma+\alpha \max \left\{\sigma^{\prime}, \frac{\sigma^{\prime}}{\alpha}\right\} \geq-\sigma+\max \{2 \alpha \sigma, 2 \sigma\} \geq \sigma
$$

Assim, $x_{1}(2)>\sigma$, o que é uma contradição.

Isto garante a existência de $\tau_{1} \in(0, \hat{t}]$ de acordo com o enunciado. $\diamond$

Lema 3.9. Admitamos as hipóteses do Lema 3.8 e sejam $k$ e $\sigma$ definidos em (3.17) e (3.20), respectivamente. Se, para $\phi \in K$, a solução $x(t ; \phi)$ de $(E)$ satisfaz $x_{1}\left(\tau_{1} ; \phi\right)>$ $\sigma, x_{2}\left(\tau_{1} ; \phi\right) \geq k$ para algum $\tau_{1} \geq 0$, então $\exists \tau_{2}=\tau_{2}(\phi)>0$ tal que $x_{1}(t ; \phi)>\sigma e^{-1}$ para $t \in\left[\tau_{1}, \tau_{2}\right], 0<x_{2}\left(\tau_{2} ; \phi\right)<k$.

Prova: Como $x_{1}\left(\tau_{1} ; \phi\right)>\sigma$, segue de $(E a)$ que $x_{1}(t ; \phi)>\sigma e^{-1}, t \in\left[\tau_{1}, \tau_{1}+1\right]$.

Se $0<x_{2}(t ; \phi)<k$ para algum $t \in\left[\tau_{1}, \tau_{1}+1\right]$, definimos $\tau_{2}$ como sendo tal $t$.

Suponhamos, então, que $x_{2}(t ; \phi) \geq k$ para todo $t \in\left[\tau_{1}, \tau_{1}+1\right]$.

Seja $\tilde{t}=\tilde{t}(\phi) \geq \tau_{1}+1$ o instante $t$ para o qual $x_{2}(t ; \phi)=k$ e $0<x_{2}(t+\varepsilon ; \phi)<$ $k, \forall \varepsilon>0$ suficientemente pequeno.

A equação $(E a)$ e as expressões (3.18) e (3.20) implicam que

$$
x_{1}^{\prime}(t) \geq-x_{1}(t)+\sigma e^{-1}, \quad t \in\left[\tau_{1}+1, \tilde{t}+1\right]
$$

e então

$$
x_{1}(t)>\sigma e^{-1}, \forall t \in\left[\tau_{1}+1, \tilde{t}+1\right]
$$


Consequentemente, existe $\tau_{2}$ de acordo com o enunciado. $\diamond$

Lema 3.10. Admitamos as hipóteses do Lema 3.8 e sejam $k$ e $\sigma$ definidos em (3.17) e (3.20), respectivamente. Então $\exists c>0$ tal que para $\phi \in K$ e $x(t ; \phi)$ solução de $(E)$ satisfazendo $x_{1}\left(\tau_{2} ; \phi\right)>\sigma e^{-1}, 0<x_{2}\left(\tau_{2} ; \phi\right)<k$ para algum $\tau_{2} \geq 0$ se tem $x_{1}\left(t_{1} ; \phi\right) \geq c$, com $t_{1}=t_{1}(\phi)$ definido no Lema 3.2 .

Prova: Se $x_{1}(t ; \phi)>\sigma e^{-1}$ em algum intervalo $\left(t_{1}-\delta, t_{1}\right)$ definimos $c:=\sigma e^{-1}$.

Suponhamos, então, que isto não aconteça e seja $\bar{t}=\bar{t}(\phi), \tau_{2}<\bar{t}<t_{1}$ o último instante $t$ para o qual $x_{1}(t ; \phi)=\sigma e^{-1}$.

Admitindo $t_{1}>\bar{t}+1$, segue da equação $(E b)$ e de (3.16),

$$
x_{2}(t ; \phi) \leq e^{-(t-\bar{t}-1)} x_{2}(\bar{t}+1)+\alpha(-1+\eta) e^{-t} \int_{\bar{t}+1}^{t} e^{s} x_{1}(s-1 ; \phi) d s
$$

para $t \in\left[\bar{t}+1, t_{1}+1\right]$.

Como $e^{t} x_{1}(t)$ é monótona crescente em $Q_{1}$,

$$
x_{2}(t ; \phi) \leq e^{-(t-\bar{t}-1)}\left[k+\alpha(-1+\eta) \sigma e^{-1}(t-\bar{t}-1)\right] .
$$

$\mathrm{O}$ segundo membro desta desigualdade se anula no instante $\tau$ definido por

$$
\tau=\frac{k e}{\alpha(1-\eta) \sigma}+\bar{t}+1
$$

Da equação $(E a)$,

$$
x_{1}(t ; \phi) \geq \sigma e^{-(t-\bar{t}+1)}, t \in\left[\bar{t}, t_{1}+1\right]
$$

Como $t_{1} \leq \tau$, segue de (3.21) e (3.22) que $x_{1}\left(t_{1} ; \phi\right) \geq c$ onde

$$
c:=\sigma e^{\left(-2+\frac{k e}{\alpha(-1+\pi) \sigma}\right)}
$$

Se $t_{1} \leq \bar{t}+1$, a desigualdade (3.22) implica que

$$
x_{1}\left(t_{1} ; \phi\right) \geq \sigma e^{-2}>c . \diamond
$$


Lema 3.11. Seja $d>0$. Existe $c>0$ tal que se $\phi \in K,\|\phi\| \geq d$, então $x_{1}\left(t_{1} ; \phi\right) \geq c$ e $x_{1}(t ; \phi) \geq c e^{-1}$ para $t \in\left[t_{1}, t_{1}+1\right]$, com $t_{1}=t_{1}(\phi) \geq 0$ definido no Lema 3.2.

Prova: Basta provar a primeira parte do Lema, já que a segunda segue trivialmente da primeira e da equação $(E a)$.

Se $\phi_{2}(0)>0$, é consequência dos Lemas $3.8,3.9$ e 3.10 .

Se $\phi_{2}(0)=0$ então $\phi_{1}(0) \geq d e^{-1}$, já que $\|\phi\| \geq d$.

Além disso, $t_{1} \in[0,1)$ e por $(E a), x_{1}\left(t_{1} ; \phi\right) \geq d e^{-2}$. $\diamond$

O lema seguinte é parte substancial da prova do Lema 3.7.

Lema 3.12. Para qualquer constante real $d, 0<d<\alpha M$, $m=\inf \left\{\left|<\sigma, x_{t}>\right| \mid x_{t}=x_{t}(\cdot ; \phi), \phi \in K, d \leq\|\phi\| \leq \alpha M, 0 \leq t \leq t_{1}(\phi)+1\right\}>0$.

Prova: Tomando a solução $v=(1,-i)$ da equação $v \Delta(\lambda)=0, \sigma(s)=(1,-i) e^{-\lambda s}$, $s \in[0,1]$ e $\eta(\theta)=\left(\eta_{i j}(\theta)\right)$ satisfazendo (3.12) segue de (3.13),

$$
<\sigma, x_{t}>=x_{1}(t)-i x_{2}(t)+\alpha \int_{-1}^{0} e^{-\lambda(\xi+1)}\left[x_{2}(t+\xi)+i x_{1}(t+\xi)\right] d \xi
$$

Suponhamos $m=0$. Então existem sequências $\phi_{n} \in K, d \leq\left\|\phi_{n}\right\| \leq \alpha M, t_{n} \in$ $\left[0, t_{1}\left(\phi_{n}\right)+1\right], n=1,2, \ldots$ tais que se $x\left(t ; \phi_{n}\right)=x_{n}(t)=\left(x_{1 n}(t), x_{2 n}(t)\right)$,

$$
<\sigma, x_{n_{t n}}>=x_{1 n}\left(t_{n}\right)-i x_{2 n}\left(t_{n}\right)+\alpha \int_{-1}^{0} e^{-\lambda(\xi+1)}\left[x_{2 n}\left(t_{n}+\xi\right)+i x_{1 n}\left(t_{n}+\xi\right)\right] d \xi
$$

tende a zero, quando $n$ tende a $\infty$.

Tomando $\lambda=a+b i$ com $a>0,0<b<\frac{\pi}{2}$, a expressão acima é equivalente a

$$
\begin{aligned}
& \left.<\sigma, x_{n_{t n}}>=x_{1 n\left(t_{n}\right.}\right)+\alpha \int_{-1}^{0} e^{-a(\xi+1)}\left[x_{2 n}\left(t_{n}+\xi\right) \cos b(\xi+1)+x_{1 n}\left(t_{n}+\xi\right) \operatorname{sen} b(\xi+1)\right] d \xi \\
& +i\left\{-x_{2 n}\left(t_{n}\right)+\alpha \int_{-1}^{0} e^{-a(\xi+1)}\left[x_{1 n}\left(t_{n}+\xi\right) \cos b(\xi+1)-x_{2 n}\left(t_{n}+\xi\right) \operatorname{sen} b(\xi+1)\right] d \xi\right\}
\end{aligned}
$$


Como $d \leq\left\|\phi_{n}\right\| \leq \alpha M$, segue da prova do Lema 3.1 que $\left|x_{n}(t)\right| \leq \alpha M$ para $t \geq-1$ e para qualquer $n$.

Assim, $x_{n}\left(t_{n}\right)$ é convergente, tomando uma subsequência, se necessário.

$\operatorname{Seja}\left(L_{1}, L_{2}\right)=\lim _{n \rightarrow \infty}\left(x_{1 n}\left(t_{n}\right), x_{2 n}\left(t_{n}\right)\right)$.

Pelos Lemas 3.8 à 3.11, podemos afirmar que $L_{1}$ e $L_{2}$ não são simultaneamente nulos.

Se $L_{1}>0, L_{2} \geq 0, \Re<\sigma, x_{n_{t_{n}}}>$ fica positiva e longe do zero para $n$ suficientemente grande e se $L_{1}=0, L_{2}>0, \Im<\sigma, x_{n_{t_{n}}}>$ fica negativa e longe do zero para $n$ suficientemente grande, e temos uma contradição, em ambos os casos.

Analisemos, então, o caso $L_{2}<0$.

É claro que $L_{1}>0$ já que, para $n$ suficientemente grande, $t_{1}\left(\phi_{n}\right) \leq t_{n} \leq t_{1}\left(\phi_{n}\right)+1$ e pelo Lema $3.11, x_{1}\left(t_{n} ; \phi_{n}\right) \geq c e^{-1}$.

Assim, na expressão (3.23), para $n$ suficientemente grande,

$$
\left\{\begin{array}{l}
x_{1 n}\left(t_{n}\right)>\frac{L_{1}}{2} \\
x_{2 n}\left(t_{n}\right)<\frac{L_{2}}{2}
\end{array}\right.
$$

$p_{n}(\xi)=x_{2 n}\left(t_{n}+\xi\right)+i x_{1 n}\left(t_{n}+\xi\right), \xi \in[-1,0]$, é uma curva no plano complexo no setor $r e^{i \theta} \operatorname{com} 0 \leq \theta \leq \pi, r \geq 0$ e $e^{-\lambda(\xi+1)}, \xi \in[-1,0]$, está situada no setor $z=r e^{i \theta}$ $\operatorname{com} r \geq e^{-a} \mathrm{e} \frac{-\pi}{2}<-b \leq \theta \leq 0$.

0 integrando $e^{-\lambda(\xi+1)} p_{n}(\xi)$ tem argumento $\theta_{n}(\xi),-\frac{\pi}{2}<-b \leq \theta_{n}(\xi) \leq \pi$.

Tomando

$$
\tilde{\theta}_{n}=\max \left\{\arg e^{-\lambda(\xi+1)} p_{n}(\xi) \mid \xi \in[-1,0]\right\}
$$

prova-se que a curva $e^{-\lambda(\xi+1)} p_{n}(\xi), \xi \in[-1,0]$, está situada inteiramente no semiplano determindo pela reta com coeficiente $\tilde{\theta}_{n}$, o qual contém o semi-eixo real positivo.

Se $0 \leq \tilde{\theta_{n}} \leq \frac{\pi}{2}$, então

$$
<\sigma, x_{n_{t_{n}}}>\nrightarrow 0
$$


já que $e^{-\lambda(\xi+1)} p_{n}(\xi)$ tem parte real não negativa e vale (3.24).

Se $\frac{\pi}{2}<\tilde{\theta}_{n}<\pi$, definindo $\omega_{n}=\frac{\pi}{2}-\tilde{\theta}_{n}$, o mesmo argumento anterior implica que

$$
e^{i \omega_{n}}<\sigma, x_{n_{n}}>\nrightarrow 0
$$

do que segue o resultado. $\diamond$

Observemos que vale a mesma afirmação do Lema 3.12 se substituirmos $K$ pelas rotações $R^{j} K$ e o intervalo $\left[0, t_{1}(\phi)+1\right]$ por $\left[t_{j}(\phi)+1, t_{j+1}(\phi)+1\right], j=1,2,3$, respectivamente, o que é justificado pela simetria rotacional.

Prova do Lema 3.7: Seja $0<a<\alpha M$.

0 Lema 3.12 implica que

$$
\inf \left\{\mid<\sigma, x_{t}>\left\|x_{t}=x_{t}(\cdot ; \phi), \phi \in K,\right\| \phi \|=a, 0 \leq t \leq t_{1}(\phi)+1\right\}>0 .
$$

Pelo Lema 3.11 , sabemos que $\exists d_{1}>0$ tal que

$$
\left\|x_{t_{1}(\phi)+1}(\cdot ; \phi)\right\| \geq d_{1}, \forall \phi \in K,\|\phi\|=a .
$$

Além disso, pelo Lema 3.1,

$$
\alpha M \geq\left\|x_{t_{1}(\phi)+1}(\cdot ; \phi)\right\|, \quad \forall \phi \in K,\|\phi\|=a .
$$

Então, pelo Lema 3.12,

$$
\inf \left\{\left|<\sigma, x_{t}>\right| \mid x_{t}=x_{t}(\cdot ; \phi), \phi \in K,\|\phi\|=a, 0 \leq t \leq t_{2}(\phi)+1\right\}>0 .
$$

O resultado segue por procedimento análogo nos intervalos $\left[t_{2}(\phi)+1, t_{3}(\phi)+\right.$ $1],\left[t_{3}(\phi)+1, \tau(\phi)\right] . \diamond$

Prova do Teorema 3.1: Mostremos que a aplicação $A=A_{4} o A_{3} o A_{2} o A_{1}: K \rightarrow K$, definida após a prova do Lema 3.4, tem um ponto fixo não trivial para $\alpha>\alpha_{0}$, onde $\alpha_{0}$ está especificado no enunciado do teorema. 
Precisamos apenas mostrar que $A$ verifica as hipóteses do Teorema 1.9, o que é feito por simples combinação dos resultados de lemas anteriores e observações.

Podemos, de acordo com a discussão após a prova do Lema 2.5, escolher um autovalor da equação $(L), \lambda=a+b i$, tal que $a>0$ e $0<b<\frac{\pi}{2}$.

O Lema 3.6 mostra que a aplicação $A$ de $K$ em $K$ é completamente contínua.

As observações seguintes ao Lema 3.4 nos dão que a aplicação $\phi \in K \backslash\{0\} \rightarrow$ $\tau(\phi) \in[4, \infty)$ tal que $A \phi=x_{\tau(\phi)}(\cdot ; \phi)$ é contínua e, segundo o Lema 3.5 satisfaz a hipótese (iv) do Lema 1.4.

A hipótese (iii) do Lema 1.4 é garantida pelo Lema 3.7.

Portanto, o Lema 1.4 assegura que 0 é um ponto fixo ejetivo de $A$.

Consideremos no Teorema 1.9 o cone $K$ e a aplicação $A=A_{4} o A_{3} o A_{2} o A_{1}: K \rightarrow$ $K$. Seja $N>\alpha M$, onde $M$ é um limitante para a função $F$ e, suponhamos que $\phi \in K,\|\phi\|=N$ e $A \phi=\lambda \phi$.

Então

$$
x(\tau(\phi)+\theta ; \phi)=\lambda \phi(\theta) \quad \forall \theta,-1 \leq \theta \leq 0 .
$$

Escolhamos $\theta \in[-1,0]$ para o qual $|\phi(\theta)|=N>\alpha M$.

Como na prova do Lema 3.1, segue que $\lambda<1$.

O Teorema é agora, consequência imediata do Teorema 1.9. $\diamond$ 


\section{Capítulo 4}

\section{Exemplos}

Damos neste capítulo quatro exemplos onde aplicamos os resultados vistos nos capítulos 2 e 3. No primeiro exemplo fazemos uma aplicação do Teorema 2.2. No segundo, uma aplicação dos Teoremas 2.1 e 3.1. No terceiro exemplo mostramos que a classe de funções $F$ para a qual o Teorema 3.1 é aplicado é bastante geral. Com base no exemplo 4, onde o Teorema 3.1 também é aplicado, destacamos a generalidade dessa classe apresentando um procedimento através do qual novos exemplos podem ser criados.

\section{Exemplo 1}

A equação escalar

$$
x^{\prime}(t)=-\alpha x(t-1)[1+x(t)] ; \quad \alpha>0,
$$

foi estudada, nos anos 50, por E. Wright [9], a partir de quando muito esforço foi dedicado para este tipo de problema e inúmeras aplicações foram feitas, com significante ênfase em ciências biológicas.

Um estudo sobre as bifurcações de Hopf $\mathbf{e}$ a existência de soluções periódicas não nulas dessa equação pode ser encontrado em [4, capítulo 11, §4].

Se $x(\phi, \alpha)$ é a solução dessa equação por $\phi$ e se $\phi(0)>-1$, podemos provar que $x(\phi, \alpha)(t)>-1, t \geq 0$. 
Motivados pelo estudo feito por $E$. Wright, analisemos a seguinte equação planar:

$$
\left\{\begin{array}{l}
y_{1}^{\prime}(t)=\alpha y_{2}(t-1)\left[1+y_{1}(t)\right] \\
y_{2}^{\prime}(t)=-\alpha y_{1}(t-1)\left[1+y_{2}(t)\right], \quad \alpha>0 .
\end{array}\right.
$$

Se $y(\cdot ; \phi, \alpha)=\left(y_{1}(. ; \phi, \alpha), y_{2}(. ; \phi, \alpha)\right)$ é solução de $(4.1)$ por $\phi=\left(\phi_{1}, \phi_{2}\right)$ e se $\phi_{j}(0)>-1, j=1,2$, podemos provar que $y_{j}(t ; \phi, \alpha)>-1, t \geq 0, j=1,2$. Em outras palavras, o conjunto $\left\{\phi \in C \mid \phi_{j}(\theta)>-1, j=1,2,-1 \leq \theta \leq 0\right\}$ é positivamente invariante sob o operador solução $T(t), t \geq 0$.

De fato, mostremos que sob as hipóteses acima, $y_{1}(t)>-1$ para $t \geq 0$.

Multiplicando a primeira equação de (4.1), pelo fator integrante $e^{-\alpha \int_{0}^{t} y_{2}(s-1) d s}$, obtemos:

$$
\left[\left(1+y_{1}(t)\right) e^{-\alpha \int_{0}^{t} y_{2}(s-1) d s}\right]^{\prime}=0 .
$$

Uma integração de 0 à $t$ nos leva à:

$$
y_{1}(t)=-1+\left(1+y_{1}(0)\right) e^{\alpha \int_{0}^{t} y_{2}(s-1) d s},
$$

ou seja, $y_{1}(t)>-1$ para $t \geq 0$.

Podemos mostrar de modo inteiramente análogo que $y_{2}(t)>-1, t \geq 0$.

Considerando a solução de (4.1), $\left(y_{1}(t), y_{2}(t)\right)$, restrita ao aberto $\left\{\left(y_{1}, y_{2}\right) \in \mathbf{R}^{2} \mid\right.$ $\left.y_{1}, y_{2}>-1\right\}$, faz sentido a seguinte mudança de variáveis:

$$
x_{i}=\ln \left(1+y_{i}\right), i=1,2,
$$

que transforma (4.1) em:

$$
\left\{\begin{array}{l}
x_{1}^{\prime}(t)=\alpha\left[e^{x_{2}(t-1)}-1\right] \\
x_{2}^{\prime}(t)=-\alpha\left[e^{x_{1}(t-1)}-1\right]
\end{array}\right.
$$

Observemos que (4.2) é uma equação da forma $x^{\prime}(t)=\alpha F(x(t-1)), \alpha>0$ com

$$
\begin{array}{lr}
F_{1}\left(x_{1}, x_{2}\right)= & e^{x_{2}}-1 \\
F_{2}\left(x_{1}, x_{2}\right)= & -e^{x_{1}}+1
\end{array}
$$


Temos que $x_{2} F_{1}\left(x_{1}, x_{2}\right)=x_{2}\left(e^{x_{2}}-1\right)>0$, se $x_{2} \neq 0$, já que $e^{x_{2}}>1$ se $x_{2}>0$ e $e^{x_{2}}<1$ se $x_{2}<0$.

Do mesmo modo, temos que $x_{1} F_{2}\left(x_{1}, x_{2}\right)=x_{1}\left(-e^{x_{1}}+1\right)<0$, se $x_{1} \neq 0$.

Além disso $\frac{\partial F_{1}}{\partial x_{2}}\left(x_{1}, x_{2}\right)=e^{x_{2}}$ e $\frac{\partial F_{2}}{\partial x_{1}}\left(x_{1}, x_{2}\right)=-e^{x_{1}}$. Portanto, $\delta_{1}=\frac{\partial F_{1}}{\partial x_{2}}(0,0)=1$ e $\delta_{2}=\frac{\partial F_{2}}{\partial x_{1}}(0,0)=-1$. Assim, a hipótese $(H .1)$ está satisfeita. Logo, de acordo com o Teorema 2.2, a equação (4.2) tem uma bifurcação de Hopf em cada valor $\alpha=k \pi, k=1,2, \ldots$.

Como a aplicação

$$
\begin{aligned}
U:\left\{\left(y_{1}, y_{2}\right) \in \mathbf{R}^{2} \mid y_{1}, y_{2}>-1\right\} & \longrightarrow \mathbf{R}^{2} \\
U_{1}\left(y_{1}, y_{2}\right) & =\ln \left(1+y_{1}\right) \\
U_{2}\left(y_{1}, y_{2}\right) & =\ln \left(1+y_{2}\right)
\end{aligned}
$$

é um difeomorfismo e $y(t)=U^{-1} x(t)$ temos que o mesmo resultado vale para (4.1).

\section{Exemplo 2}

Consideremos a seguinte equação planar:

$$
\left\{\begin{array}{l}
y_{1}^{\prime}(t)=\left[-\ln \left(1+y_{1}(t)\right)+\alpha \arctan y_{2}(t-1)\right]\left(1+y_{1}(t)\right) \\
y_{2}^{\prime}(t)=\left[-\ln \left(1+y_{2}(t)\right)-\alpha \arctan y_{1}(t-1)\right]\left(1+y_{2}(t)\right), \alpha>0
\end{array}\right.
$$

Como no exemplo 1 , podemos provar que o conjunto $\left\{\phi \in C \mid \phi_{j}(\theta)>-1, j=\right.$ $1,2,-1 \leq \theta \leq 0\}$ é positivamente invariante sob o operador solução $T(t), t \geq 0$.

Considerando a solução de (4.3), $\left(y_{1}(t), y_{2}(t)\right)$, restrita ao aberto $\left\{\left(y_{1}, y_{2}\right) \in \mathbf{R}^{2} \mid\right.$ $\left.y_{1}, y_{2}>-1\right\}$, a mesma mudança de variáveis:

$$
x_{i}=\ln \left(1+y_{i}\right), i=1,2,
$$

transforma (4.3) em: 


$$
\left\{\begin{array}{l}
x_{1}^{\prime}(t)=-x_{1}(t)+\alpha \arctan \left(e^{x_{2}(t-1)}-1\right) \\
x_{2}^{\prime}(t)=-x_{2}(t)-\alpha \arctan \left(e^{x_{1}(t-1)}-1\right), \quad \alpha>0
\end{array}\right.
$$

Observemos que (4.4) é uma equação planar da forma

$$
x^{\prime}(t)=-x(t)+\alpha F(x(t-1)), \alpha>0
$$

com

$$
\begin{aligned}
& F_{1}\left(x_{1}, x_{2}\right)=\arctan \left(e^{x_{2}}-1\right) \\
& F_{2}\left(x_{1}, x_{2}\right)=-\arctan \left(e^{x_{1}}-1\right)
\end{aligned}
$$

É fácil ver que a hipótese (H.1) está satisfeita. Logo, de acordo com o Teorema 2.1 , existe uma sequência $\alpha_{0}<\alpha_{1}<\ldots \rightarrow \infty$ tal que a equação (4.4) tem uma bifurcação de Hopf em $\alpha=\alpha_{k}, k=0,1, \ldots$.

Além disso, $F\left(x_{1}, x_{2}\right)=\left(\arctan \left(e^{x_{2}}-1\right),-\arctan \left(e^{x_{1}}-1\right)\right)$ é limitada e, então, pelo Teorema 3.1, se $\alpha_{0}=\left(1+b^{2}\right)$ sen $b, \operatorname{com} b$ univocamente definido por $b \tan b=1$ em $\left[0, \frac{\pi}{2}\right]$, temos que para todo $\alpha>\alpha_{0}$ a equação (4.4) tem uma solução periódica não trivial $x(\cdot ; \phi), \phi \in K$ com período $T>4$, onde $K$ é o cone definido em (3.1).

Observando que $y(t)=U^{-1} x(t)$ onde $U$ é o difeomorfismo definido no exemplo 1 , temos que os mesmos resultados estabelecidos para a equação (4.4) valem também para (4.3).

\section{Exemplo 3}

Uma análise cuidadosa mostra que a classe de funções $F$ para a qual o Teorema 3.1 é aplicado é bastante geral. Em primeiro lugar observemos que, retirando a condição de limitação, as hipóteses são obviamente verificadas no caso linear, $\tilde{F}(x)=$ $\left(x_{2},-x_{1}\right)$, no qual a equação $(E)$ é reduzida ao sistema:

$$
\left\{\begin{array}{l}
x_{1}^{\prime}(t)=-x_{1}(t)+\alpha x_{2}(t-1) \\
x_{2}^{\prime}(t)=-x_{2}(t)-\alpha x_{1}(t-1)
\end{array}\right.
$$


Isto sugere um modo de criar exemplos pelo truncamento da função $\tilde{F}$. Este processo pode ser realizado preservando qualquer grau de regularidade. Contudo, podemos seguramente ignorar isto, uma vez que a diferenciabilidade $C^{3}$ requerida para $F$ é apenas necessária perto da origem, onde os termos mais baixos da expansão de Taylor dominam.

O exemplo que passamos a construir faz uso das idéias acima.

Seja $F$ a função definida como segue:

$$
\begin{array}{ll}
F_{j}(x)=\tilde{F}_{j}(x), & \text { se }\left|x_{i}\right| \leq 1, i, j=1,2, \quad i \neq j \\
F_{1}(x)=\frac{x_{2}}{\left|x_{2}\right|}, & \text { se }\left|x_{2}\right| \geq 1 \\
F_{2}(x)=-\frac{x_{1}}{\left|x_{1}\right|}, & \text { se }\left|x_{1}\right| \geq 1 .
\end{array}
$$

Neste exemplo, temos que além da hipótese $(H .1)$ estar satisfeita, $F$ é limitada, logo vale (H.2).

Portanto, o Teorema 3.1 é válido.

Observemos que nos exemplos anteriores, cada componente de $F$ é independente de uma das variáveis $x_{1}$ e $x_{2}$.

No exemplo a seguir as componentes de $F$ dependem efetivamente das duas variáveis $x_{1}$ e $x_{2}$ e $F$ satisfaz as hipóteses $(H .1)$ e (H.2).

\section{Exemplo 4:}

$$
\left\{\begin{array}{l}
x_{1}^{\prime}(t)=-x_{1}(t)+\frac{2 \alpha e^{x_{1}(t-1)+x_{2}(t-1)} \arctan x_{2}(t-1)}{1+e^{x_{1}(t-1)+x_{2}(t-1)}} \\
x_{2}^{\prime}(t)=-x_{2}(t)-\frac{2 \alpha e^{x_{1}(t-1)-x_{2}(t-1)} \arctan x_{1}(t-1)}{1+e^{x_{1}(t-1)-x_{2}(t-1)}}, \alpha>0
\end{array}\right.
$$

A equação (4.5) é, obviamente, do tipo $(E)$, onde $F$ foi construída segundo o critério geral abaixo que, na verdade, descreve um procedimento para a criação de novos exemplos.

Se $G_{j}(x)$ é uma função de classe $C^{3}$, limitada, positiva, $G_{j}(0,0)=1, j=1,2$, e se $\tilde{F}$ satisfaz as hipóteses do Teorema 3.1, então a função $F$ cujas componentes 
são $F_{j}=\tilde{F}_{j} G_{j}, j=1,2$, satisfaz as hipóteses (H.1) e (H.2), garantindo a validade dos teoremas 2.1 e 3.1 para a equação (E).

De fato, temos que: $x_{2} F_{1}(x)=x_{2} \tilde{F}_{1}(x) G_{1}(x)>0$, se $x_{2} \neq 0$, já que $\tilde{F}$ satisfaz (H.1) e $G_{1}$ é positiva.

Do mesmo modo, $x_{1} F_{2}(x)=x_{1} \tilde{F}_{2}(x) G_{2}(x)<0$, se $x_{1} \neq 0$.

Além disso,

$$
\delta_{1}=\frac{\partial F_{1}}{\partial x_{2}}(0,0)=\tilde{F}_{1}(0,0) \frac{\partial G_{1}}{\partial x_{2}}(0,0)+\frac{\partial \tilde{F}_{1}}{\partial x_{2}}(0,0) G_{1}(0,0)=1
$$

uma vez que $\tilde{F}_{1}(0,0)=0, \frac{\partial \tilde{F}_{1}}{\partial x_{2}}(0,0)=1, G_{1}(0,0)=1$ e $\frac{\partial G_{1}}{\partial x_{2}}(0,0) \in \mathbf{R}$.

Do mesmo modo,

$$
\delta_{2}=\frac{\partial F_{2}}{\partial x_{1}}(0,0)=-1
$$

Assim, a hipótese ( $H .1)$ é válida.

Como $F_{j}=\tilde{F}_{j} G_{j}, \quad j=1,2$, é imediato que $F$ é limitada e portanto satisfaz (H.2). 


\section{Referências}

1 BAPTISTINI, M.T.Z. Soluções periódicas de uma equação diferencial no plano com retardamento e continuação global. São Carlos, 1990. p.26-34. (Tese Doutorado - Instituto de Ciências Matemáticas de São Carlos, da Universidade de São Paulo).

2 BROWDER, F. A further generalization of the Schauder fixed point theorem. Duke Math., 32: 575-578, 1965.

3 CHOW, S.N. \& HALE, J.K. Periodic solutions of autonomous equations. J. Math. Anal. Appl., 66: 495-506, 1978.

4 HALE, J.K. Theory of functional differential equations. $11^{\underline{a}}$ edição. New York, Springer-Verlag, 1977. 365p.

5 JONES, G. The existence of periodic solutions of $f^{\prime}(x)=-\alpha f(x-1)[1+f(x)]$. J.Math. Anal. Appl., 5: 435-450, 1962.

6 NUSSBAUM, R. Periodic solutions of nonautonomous functional differential equations. In: FUNCTIONAL DIFFERENTIAL EQUATIONS and APPROXIMATIONS of FIXED POINTS, eds H.O. Peitgen and H.O. Walther, Lecture Notes in Mathematics 730 (Berlin: Springer, 1979).

7 ONUCHIC, N. Equações diferenciais com retardamento. In: COLÓQUIO BRASILEIRO DE MATEMÁTICA, 8o, Poços de Caldas, 1971. L Livro... Poços de Caldas, IMPA, 1971. 85p.

8 TÁBOAS, P.Z. Periodic solutions of a planar delay equation. Proc. Royal Soc. Edinburgh, 116A: 85-101, 1990.

9 WRIGHT, E. A nonlinear difference-differential equation. J. Reine Angew Math., 194: 66-87, 1955. 